\title{
T follicular regulatory cells and IL-10 promote food antigen-specific IgE
}

\author{
Markus M. Xie, ${ }^{1}$ Qiang Chen, ${ }^{1}$ Hong Liu, ${ }^{1}$ Kai Yang, ${ }^{2}$ Byunghee Koh, ${ }^{2}$ Hao Wu, ${ }^{1}$ Soheila J. Maleki, ${ }^{3}$ Barry K. Hurlburt, ${ }^{3}$ \\ Joan Cook-Mills, ${ }^{2}$ Mark H. Kaplan, ${ }^{2}$ and Alexander L. Dent ${ }^{1}$
}

'Department of Microbiology and Immunology and ${ }^{2}$ Department of Pediatrics, Indiana University School of Medicine, Indianapolis, Indiana, USA. ${ }^{3}$ Agricultural Research Service, United States Department of Agriculture, Baton Rouge, Louisiana, USA.

\begin{abstract}
Food allergies are a major clinical problem and are driven by IgE antibodies (Abs) specific for food antigens (Ags). T follicular regulatory (Tfr) cells are a specialized subset of FOXP3 ${ }^{+} \mathrm{T}$ cells that modulate $\mathrm{Ab}$ responses. Here, we analyzed the role of $\mathrm{Tfr}$ cells in regulating Ag-specific IgE using a peanut-based food allergy model in mice. Peanut-specific IgE titers and anaphylaxis responses were significantly blunted in Tfr cell-deficient Foxp3-Cre Bcl $6^{f / f l}$ mice. Loss of Tfr cells led to greatly increased nonspecific IgE levels, showing that Tfr cells have both helper and suppressor functions in IgE production in the germinal center (CC) that work together to facilitate the production of Ag-specific IgE. Foxp3-Cre Pten ${ }^{f / f l}$ mice with augmented Tfr cell responses had markedly higher levels of peanut-specific IgE, revealing an active helper function by Tfr cells on Ag-specific IgE. The helper function of Tfr cells for IgE production involves IL-10, and the loss of IL-10 signaling by B cells led to a severely curtailed peanutspecific IgE response, decreased GCB cell survival, and loss of GC dark zone B cells after peanut sensitization. We thus reveal that Tfr cells have an unexpected helper role in promoting food allergy and may represent a target for drug development.
\end{abstract}

\section{Introduction}

Antibodies (Abs) that bind to antigen (Ag) with high affinity and help to eliminate pathogens and foreign substances are generated in the germinal center (GC) reaction (1-3). A specialized subset of differentiated $\mathrm{CD}^{+} \mathrm{T}$ cells, $\mathrm{T}$ follicular helper (Tfh) cells, are required in the GC reaction to help B cells generate high-affinity Abs (4). Tfh cells control the initiation as well as the overall outcome of the GC B (GCB) cell response $(5,6)$. Although Tfh cells are required to produce protective Abs, the deregulation of Tfh cells can also lead to autoimmunity, since Tfh cells can help B cells to produce self-reactive Abs (6-8). Ab responses are also modulated by $\mathrm{T}$ follicular regulatory ( $\mathrm{Tfr}$ ) cells, which develop from FOXP3 ${ }^{+}$Tregs and localize to the GC a result of CXCR5 expression (9-15). Tfr cells, like Tfh cells, are dependent on the transcriptional repressor protein Bcl6 for their development, but unlike Tfh cells, Tfr cells also express the canonical Treg master regulatory transcription factor FOXP3 (9-14). Tfr cells have usually been found to act as repressor cells in the GC $(9-11,14)$. More recently, a Tfr-deficient mouse model, in which the $B c l 6$ gene was specifically deleted in $\mathrm{FOXP3}^{+} \mathrm{T}$ cells $\left(\mathrm{Bcl}^{f / f l} \mathrm{Foxp} 3-\mathrm{Cre}\right.$, referred to hereafter as Bcl6FC mice), was used by us and others to study Tfr cell function $(12,16-19)$. This mouse model has helped to show that Tfr cells can have a helper function in the GC and Ab response (15).

The increase in atopic and allergic diseases over the past 30 years is of epidemic proportions $(20,21)$. IgE mediates immediate hypersensitivity to allergens in atopic patients $(22,23)$. Despite its

Conflict of interest: The authors have declared that no conflict of interest exists. Copyright: (C) 2020, American Society for Clinical Investigation.

Submitted: August 1, 2019; Accepted: March 26, 2020; Published: June 8, 2020.

Reference information: J Clin Invest. 2020;130(7):3820-3832.

https://doi.org/10.1172/JCI132249. importance in allergic disease, how IgE responses develop in the GC is not well understood, and there is controversy over the specific pathways involved (24-28).

Recent studies have revealed that IL-4-secreting Tfh cells are absolutely required for the development of Ag-specific IgE $(29,30)$. The discovery of Tfh cells has expanded our knowledge of allergic immune responses, but much remains unclear about the regulation of allergen-specific IgE production in the GC. Furthermore, the role of Tfr cells in the IgE pathway is not known. Here, we investigated the role of Tfr cells in IgE production using a mouse model of food allergy and found, unexpectedly, that Tfr cells positively regulated the GC and Ag-specific IgE production, in part through the production of IL-10.

\section{Results}

Production of Ag-specific IgE is dependent on both Tfr cells and the $G C$ reaction. To study the role of $\mathrm{Tfr}$ cells in regulating IgE production and IgE responses, we used the Bcl6FC mouse model, in which Tfr cells do not develop (12), as well as a widely used model of food allergy induced by intragastric (i.g.) sensitization with peanut protein plus cholera toxin (PCT) as an adjuvant (31-33). In this model (Figure 1A), high levels of peanut-specific IgE are induced and maintained in the circulation for weeks (31-33), and 4 weeks after the sensitization period, control (WT) mice produce substantial levels of peanut-specific IgE (Figure 1B). In Bcl6FC mice, the peanut-specific IgE response was almost undetectable after 4 weeks (Figure 1B). Peanut-specific IgG1 was present but significantly decreased in the Bcl6FC mice (Figure 1B). At the same time, we found that total $\operatorname{IgE}$ responses were significantly increased in Bcl6FC mice, showing a repressive effect of Tfr cells on nonspecific IgE responses (Figure $1 \mathrm{~B}$ and Supplemental Figure 1; 
A

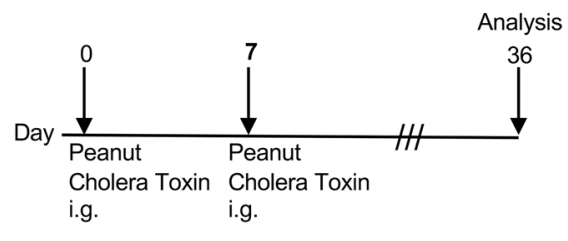

C

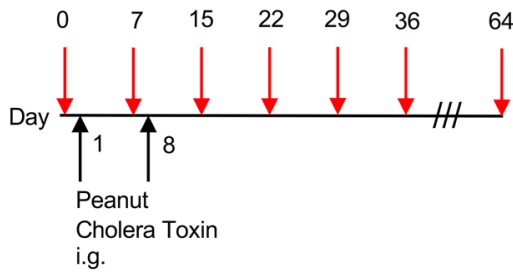

B Anti-Peanut Extract lgE

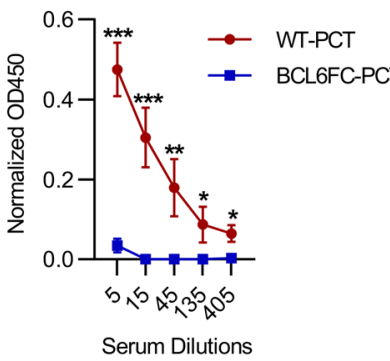

D Anti-Peanut Extract lgE

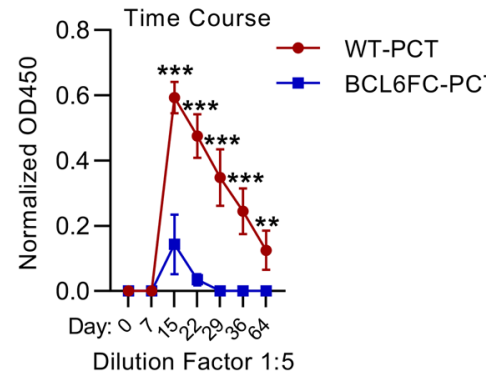

Anti-Peanut Extract lgG1

Total lgE
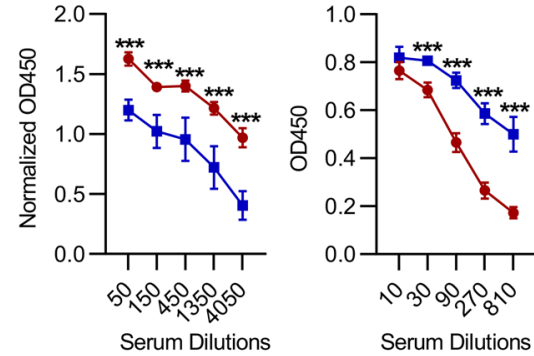

Anti-Peanut Extract IgG

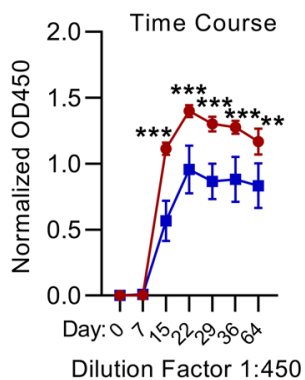

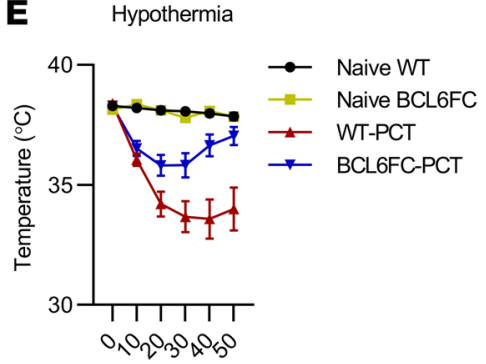

Time after challenge ( $\mathrm{min})$
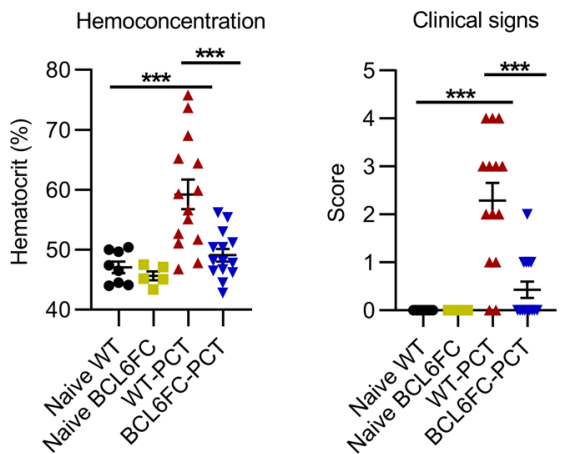

F Anti-Peanut Extract lgE

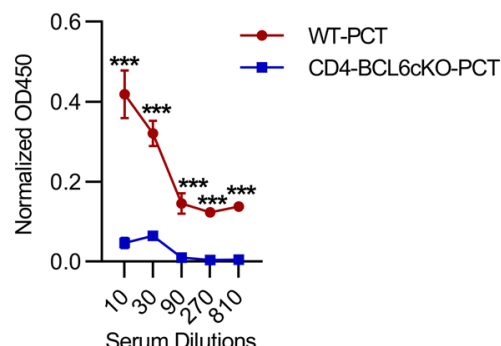

G

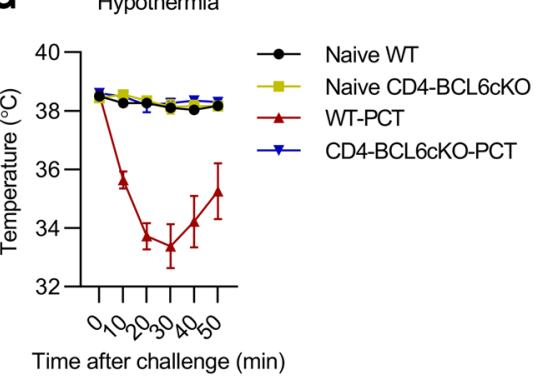

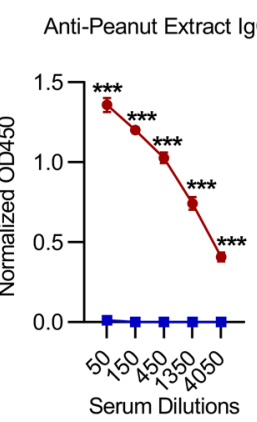
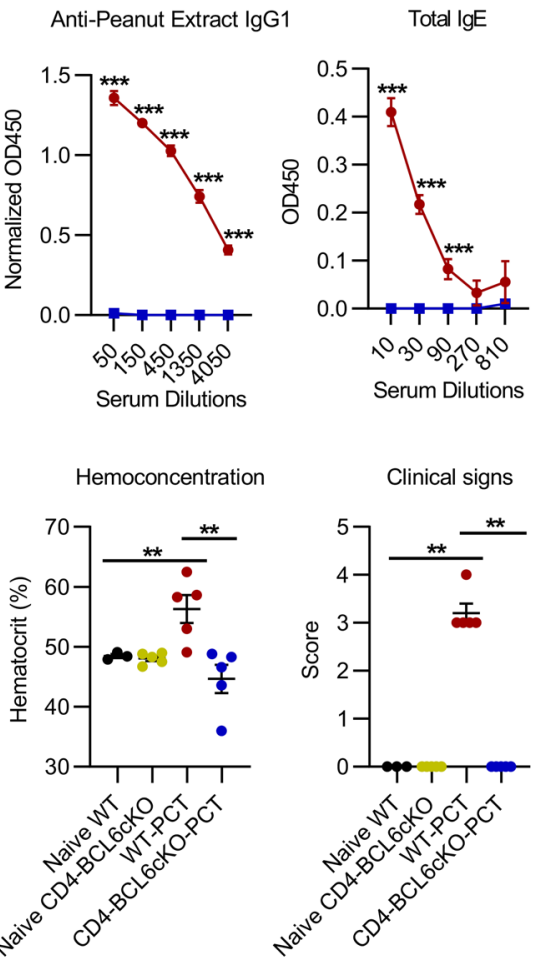

Clinical signs

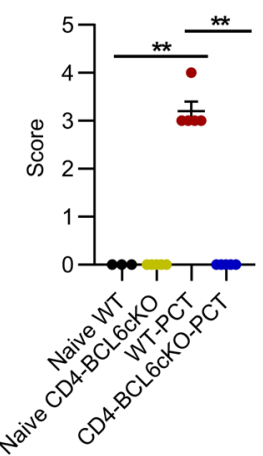

Figure 1. Lack of Tfr cells in a food allergy model leads to loss of peanut-specific IgE and decreased anaphylaxis responses. Peanut allergy was induced with 2 i.g. doses of PCT given 7 days apart, and mice were bled at various time points after sensitization. (A) Schema showing the 36-day timeline, in which serum was tested 28 days after the last sensitization for peanut-specific Abs. (B-D) Control Foxp3-Cre mice (WT) and Bcl6FC mice were sensitized as in $\mathbf{A}$, and day-36 serum was tested for peanut-specific IgE, IgG1, and total IgE (B) or at various time points during and after sensitization as indicated (red arrows in $\mathbf{C}$ ) (C and $\mathbf{D})$. Data for $\mathbf{A}$ and $\mathbf{B}$ are from 1 representative experiment of 4 experiments with 4-5 mice per group. Data for $\mathbf{C}$ and $\mathbf{D}$ are from 1 representative experiment of 2 experiments with 4-5 mice per group. (E) WT and Bcl6FC mice sensitized as in $\mathbf{A}$ were analyzed for anaphylactic responses on day 36. Nonsensitized WT and Bcl6FC mice were used as negative controls. Data for $\mathbf{E}$ were pooled from 2 experiments with 3-7 mice per group ( $n$ = 6-14). (F and G) Control Bcl $6^{f / f l}(\mathrm{WT})$ mice and CD4-Bcl6-CKO mice were sensitized as shown in $\mathbf{A}$. (F) Day-36 serum was tested for peanut-specific IgE, IgG1, and total IgE. (G) Mice were tested for anaphylaxis as described in $\mathbf{E}$. Data for $\mathbf{F}$ are from 1 representative experiment of 3 experiments with 4-5 mice per group. Data for $\mathbf{C}$ are from 1 representative experiment of 2 experiments with 3-5 mice per group. ${ }^{*} P<0.05$, ${ }^{* *} P<0.01$, and ${ }^{* *} P<0.001$, by 2-way ANOVA with Holm-Šidák multiple comparisons test (B, D, and $\mathbf{F})$ or 2-way ANOVA with Tukey's multiple comparisons test (E and $\mathbf{G}$ ). 
A

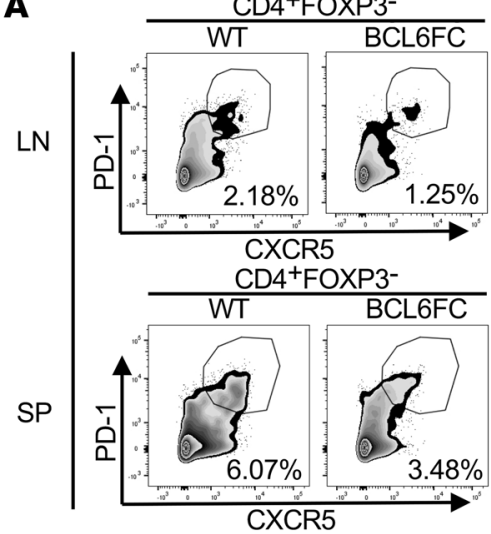

B

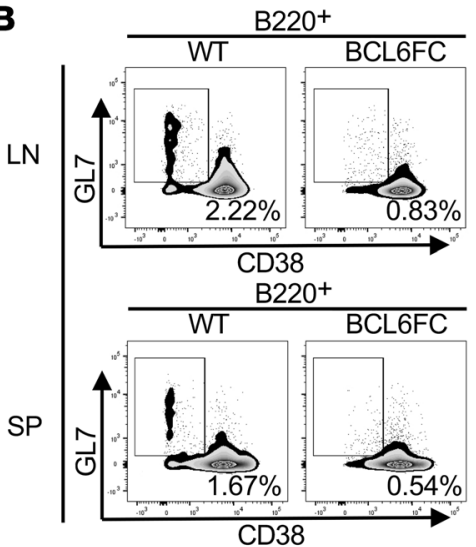

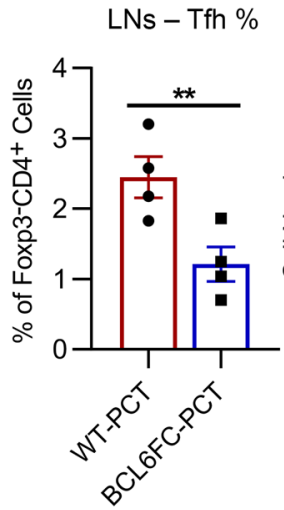

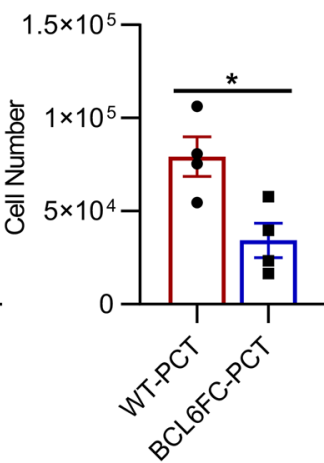

LNs - GCB \%

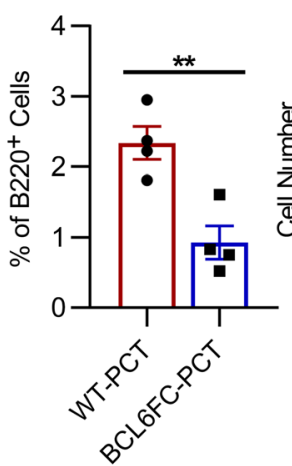

Splenic Tfh cells

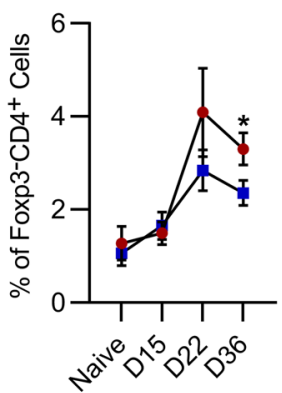

SPs - Tfh \% SPs - Tfh nos.
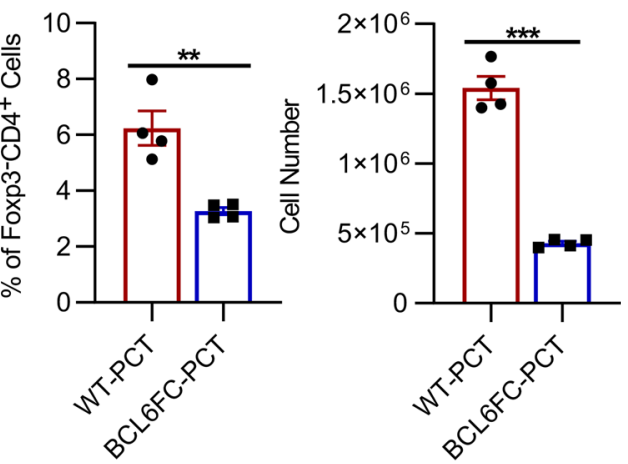

C

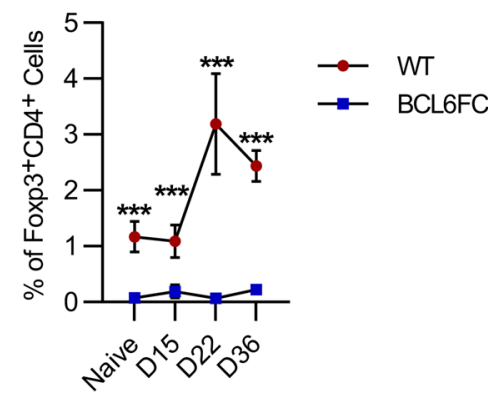

LNs - GCB nos

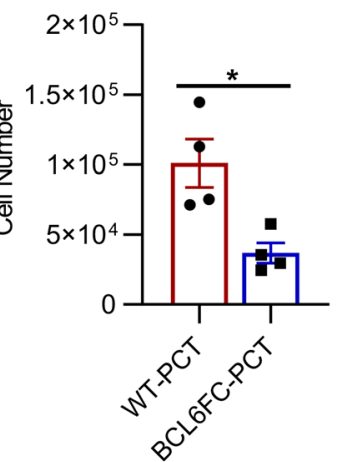

SPs - GCB \%
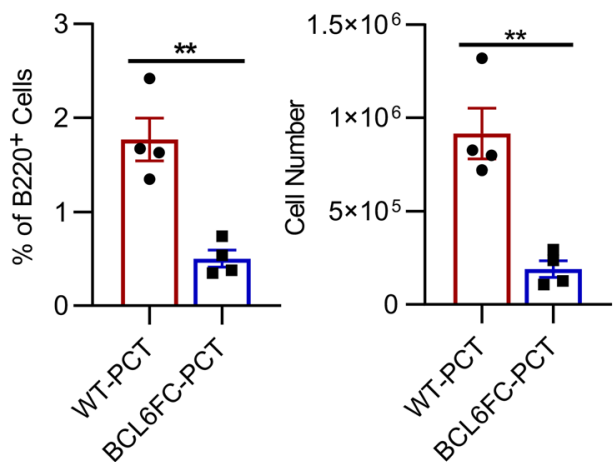

Figure 2. Tfr cells are required for normal Tfh and GCB cell numbers in a food allergy immune response. WT and Bcl6FC mice were sensitized with PCT as in Figure 1, and then on day 36, mesenteric LNs and SPs were analyzed for Tfh cells (A) and GCB cells (B) by flow cytometry. (C) Time course of the Tfr, Tfh, and GCB responses after PCT sensitization. Data for $\mathbf{C}$ were pooled from naive mice (2 experiments), day 15 (2 experiments), day 22 (2 experiments), and day 36 (8 experiments), with 4-5 mice per group $(n=8-10)$. Day 15 (D15) $=7$ days after sensitization; day 22 (D22) = 14 days after sensitization; day 36 $\left(\right.$ D36) $=28$ days after sensitization. ${ }^{*} P<0.05,{ }^{* *} P<0.01$, and ${ }^{* * *} P<0.001$, by 2-way ANOVA with Holm-Šidák multiple comparisons test (B) or 2-tailed Student's $t$ test (C).

supplemental material available online with this article; https:// doi.org/10.1172/JCI132249DS1). We then analyzed the time course of serum peanut-specific IgE and IgG1 induction and maintenance after PCT sensitization (Figure 1, C and D). We observed that in WT mice, peanut-specific IgE was induced to high levels 1 week after the second PCT sensitization (day 15) and then slowly decreased over several weeks. However, in Bcl6FC mice, the initial induction of peanut-specific IgE was much weaker and then faded to undetectable levels 2 weeks after PCT sensitization. Peanut-specific IgG1 was strongly induced and maintained at high lev- els for weeks after the PCT sensitization in both WT and Bcl6FC mice, though the levels were significantly lower in Bcl6FC mice (Figure 1D). To assess the physiological relevance of peanut-specific IgE levels, we induced anaphylaxis by giving a systemic challenge of peanut protein alone 4 weeks after mice were peanut sensitized. We found that a strong anaphylaxis response was induced in WT mice, whereas anaphylaxis was notably weaker in Bcl6FC mice (Figure 1E). The lower anaphylaxis response in Bcl6FC mice is not explained by a weaker affinity of the peanut-specific IgE, as the affinity of IgE from Bcl6FC mice for the peanut protein Ara $\mathrm{H} 3$ 
A

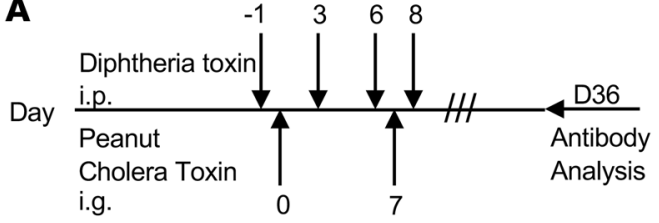

C

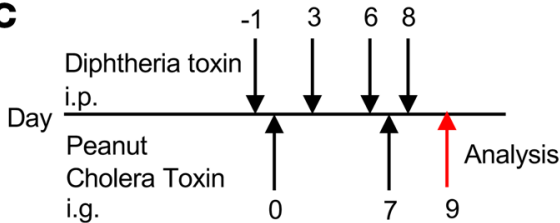

B Anti-Peanut Extract lgE

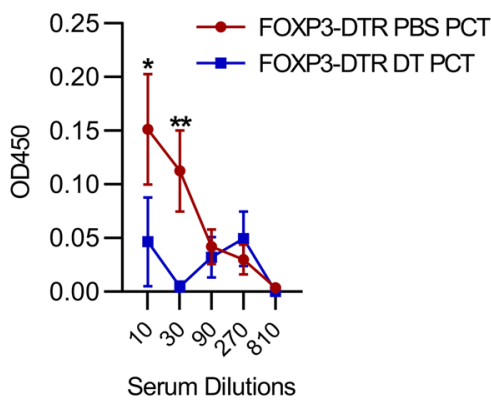

Anti-Peanut Extract lgG1

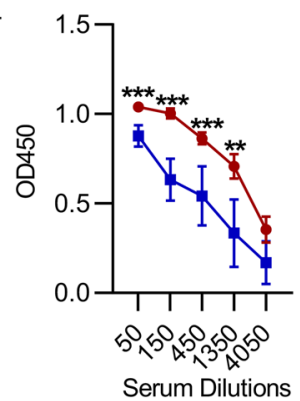

Total lgE

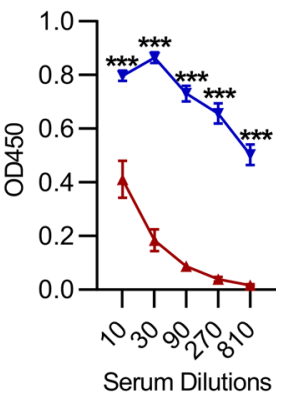

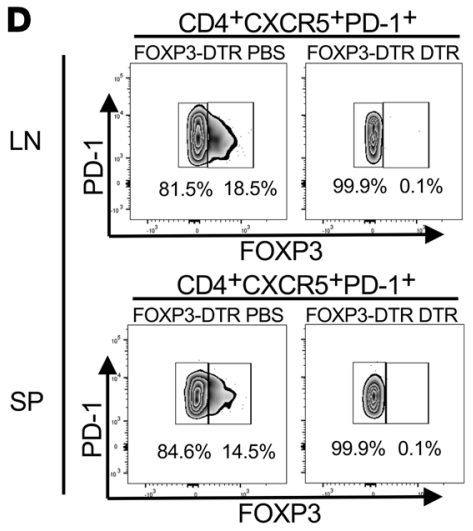
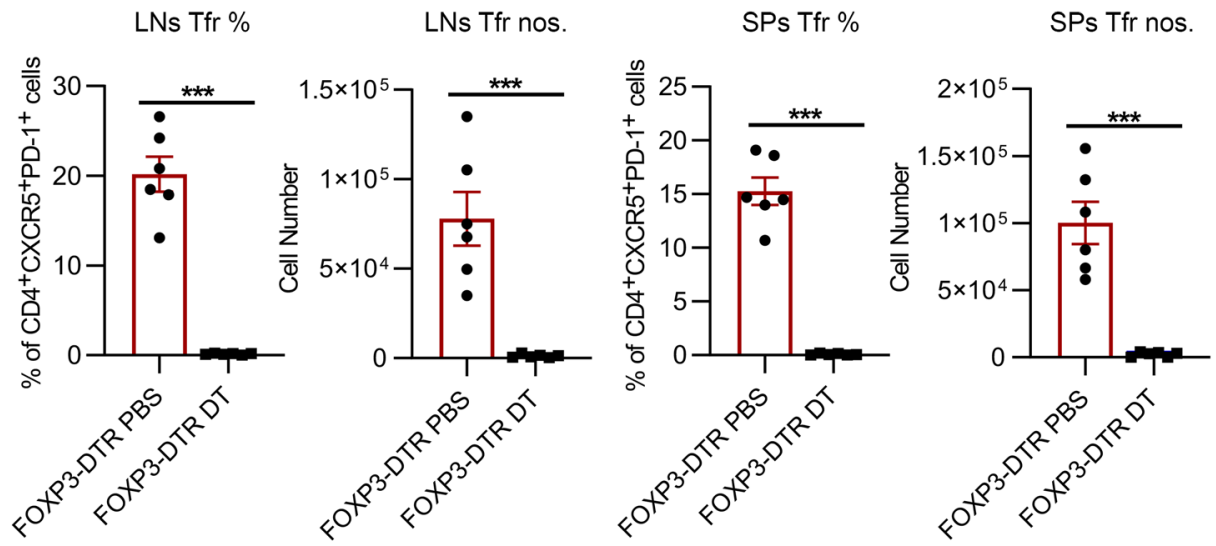

Figure 3. Total Tregs and Tfr cells are required for Ag-specific IgE and IgG1 in a food allergy response. (A and B) FOXP3-DTR mice were treated with DT as indicated to deplete Tregs, or given PBS as a control, and then sensitized with PCT as indicated and bled for serum peanut-specific IgE and IgG1 Abs on day 36. Data for $\mathbf{B}$ are from 1 representative experiment of 2 experiments with 4-5 mice per group. (C and D) FOXP3-DTR mice were treated with DT or PBS as a control, sensitized with PCT on days 0 and 7 as indicated, and then on day 9 , draining mesenteric LNs and SPs were removed for analysis of CD4 ${ }^{+}$FOXP3 ${ }^{+}$PD- ${ }^{+}$ CXCR5 ${ }^{+}$Tfr cells. Tfr cells were quantitated as the percentage of FOXP3 ${ }^{+}$cells from CD4+CXCR5 ${ }^{+} \mathrm{PD}-1^{+} \mathrm{T}$ cells and absolute numbers per $\mathrm{LN}$ or SP. Data for $\mathbf{D}$ are from 1 representative experiment of 2 experiments with 4-6 mice per group. ${ }^{*} P<0.05$, ${ }^{* *} P<0.01$, and ${ }^{* * *} P<0.001$, by 2 -tailed $t$ test (A and $\mathbf{B}$ ) or 2 -way ANOVA with Tukey's multiple comparisons test (C).

was similar to the affinity of IgE from control mice (Supplemental Figure 2). To determine whether the strong anaphylaxis response was dependent on IgE, we tested $F c \varepsilon R 1^{1 /}$ mice, which have mast cells that cannot bind IgE, in our PCT sensitization system. We found that anaphylaxis symptoms were significantly lessened in $F c \varepsilon R 1^{-1-}$ mice, indicating that IgE mediated much of the response to the peanut challenge (Supplemental Figure 2). We then tested whether the induction of peanut-specific IgE was dependent on the GC reaction by analyzing $\mathrm{Cd} 4$ - $\mathrm{Cre} B \mathrm{Bcl} 6^{f / f l}$ mice (referred to hereafter as CD4-BCL6-cKO), which lack Tfh cells and cannot mount GC reactions (34). After PCT sensitization, anti-peanut $\operatorname{IgE}$ and IgG1 responses and total IgE responses were completely ablated in these mice (Figure 1F), as well as in mice that could form GCs due to B cell-specific deletion of Bcl6 (Supplemental Figure 3). CD4-BCL6-cKO mice showed no signs of anaphylaxis when challenged systemically with peanut protein (Figure 1G), consistent with the loss of peanut-specific IgE in these mice. We obtained similar results showing Ag-specific IgE dependence on GC responses and Tfr cells when OVA was substituted for peanut protein in the food allergy model (Supplemental Figure 4), demonstrating that these results were not unique to peanut as an Ag.
Tfr cells are required to maintain GC responses over time. We wondered whether the loss of peanut-specific IgE was explained by a loss of B cells in the GCs of Bcl6FC mice and thus examined GCB, $\mathrm{Tfh}$, and Tfr cells in mesenteric lymph nodes (LNs) and spleens (SPs) to see if there was a defect in the GC reaction. In this model, both Tfh and Tfr cells from WT mice were over $90 \%$ CD45RB but Tfr cells expressed roughly half as much CXCR5 as Tfh cells (Supplemental Figure 5). Lower CXCR5 expression on Tfr cells compared with expression on Tfh cells has been observed previously with human cells (35). As expected, we found that Tfr cells were almost completely absent in Bcl6FC mice despite a robust Tfr response in WT mice on day 36 of the PCT sensitization (Supplemental Figure 6A). Unexpectedly, we observed a significant decrease in Tfh cells in Bcl6FC mice after PCT sensitization (Figure $2 \mathrm{~A}$ ) and an even larger loss of GCB cells ( $70 \%$ decrease) in Bcl6FC mice after the PCT sensitization (Figure 2B). This loss of Tfh cells in Bcl6FC mice was not due to aberrant or "leaky" deletion of Bcl6 in FOXP3 ${ }^{\text {YFP }}$-negative Tht cell precursor cells, as we did not detect significant levels of FOXP3-Cre activity in FOXP3 ${ }^{\mathrm{YFP}}$-negative $\mathrm{CD} 4^{+}$ T cells (Supplemental Figure 6, B-E). Next, we examined the time course of the GC response and noted that in WT mice, Tfr, Tfh, and 
A

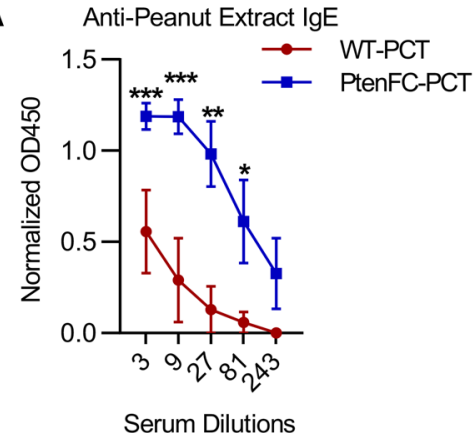

B

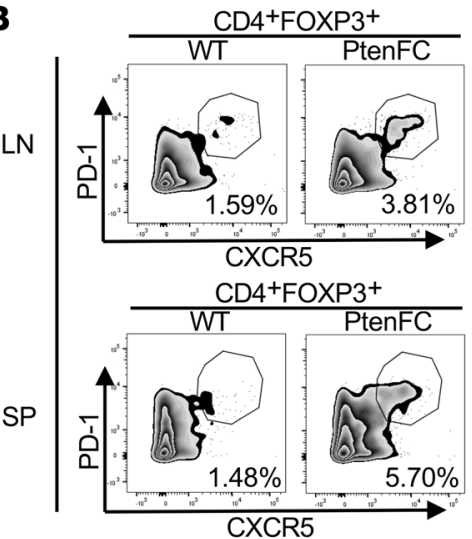

C
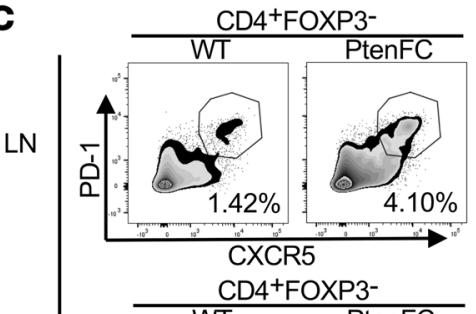

SP

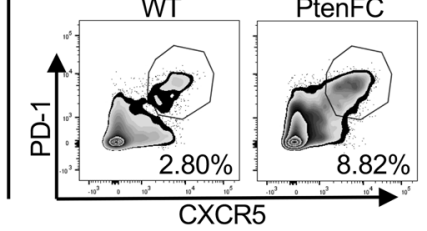

D

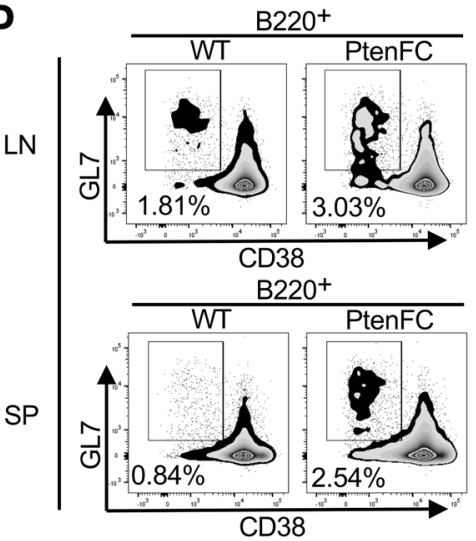

Anti-Peanut Extract IgG1

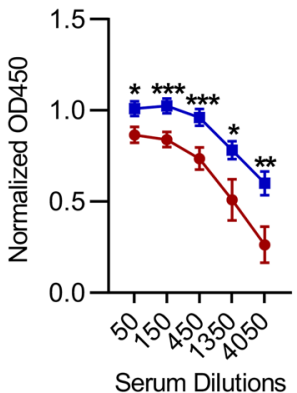

Total lgE

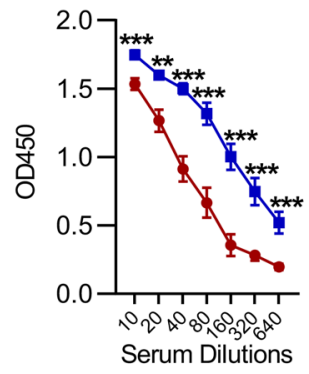

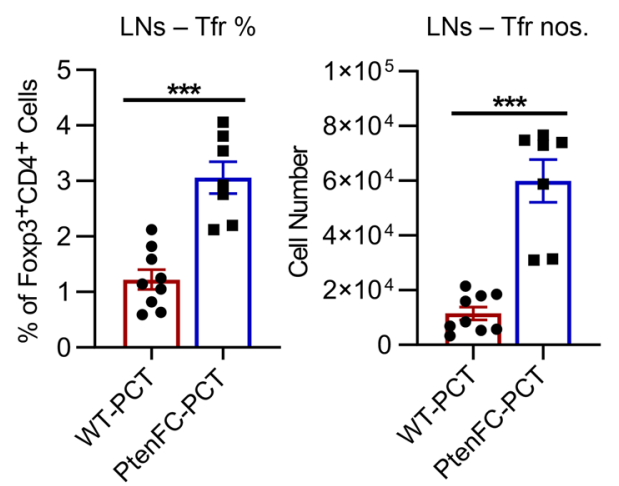
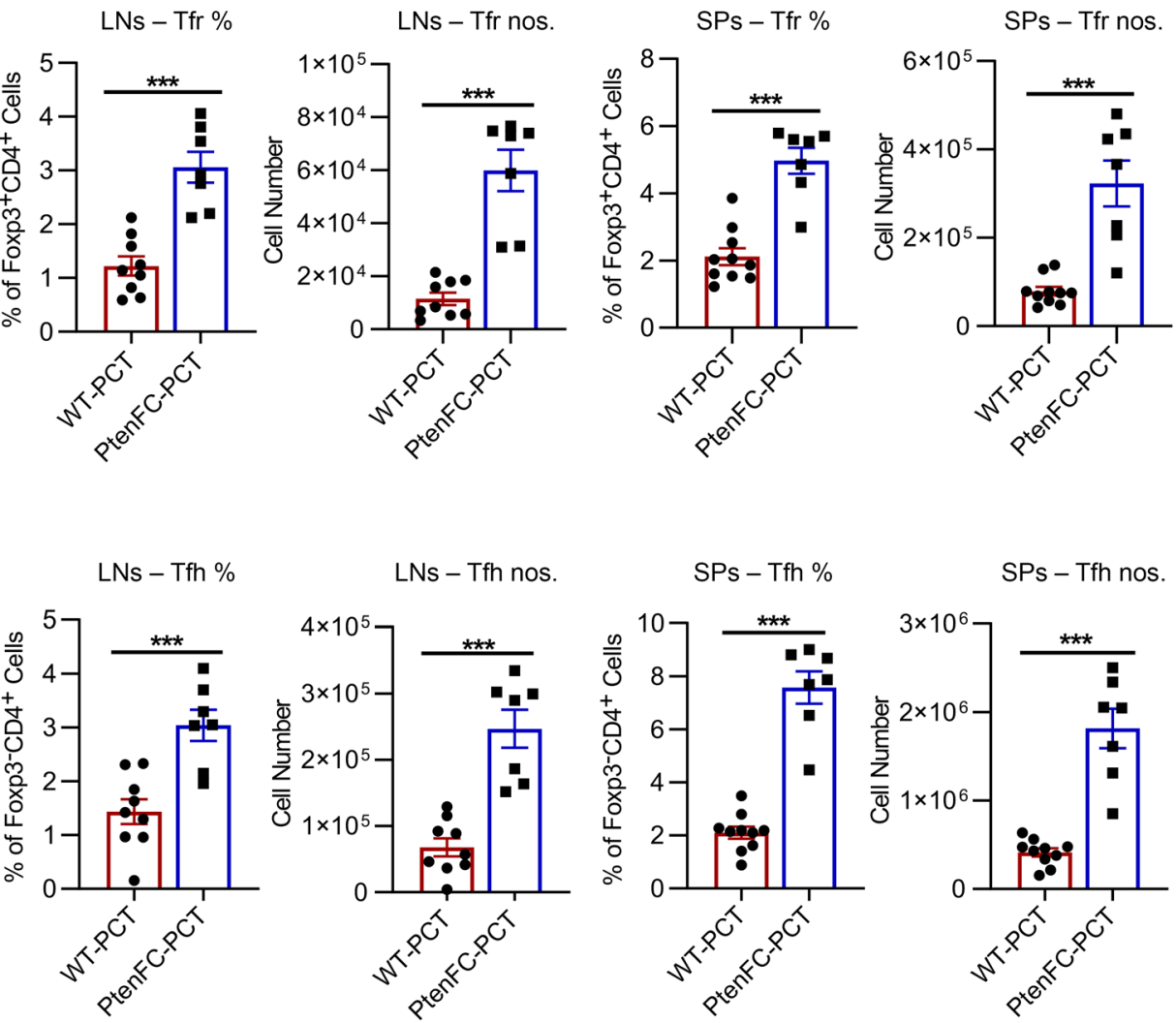

LNs - Tfh nos.

SPs - Tfh \%

SPs - Tfh nos.
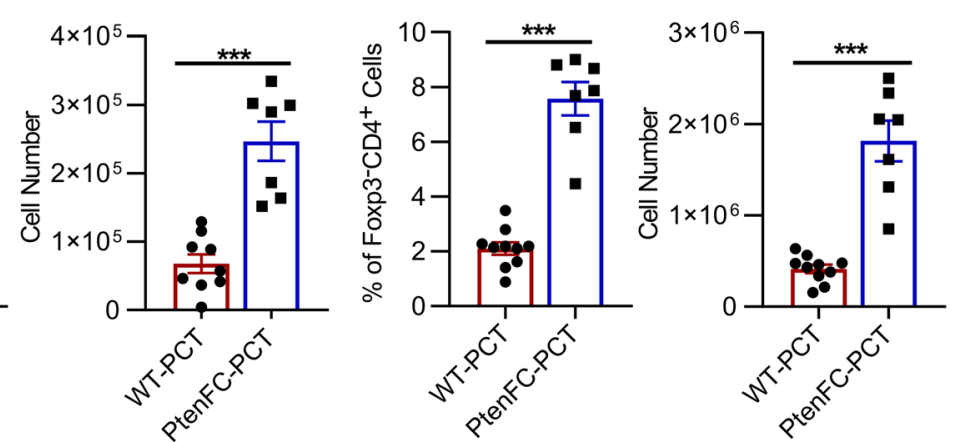

LNs - GCB \%

LNs - GCB nos.

SPs - GCB \%

SPs - GCB nos.
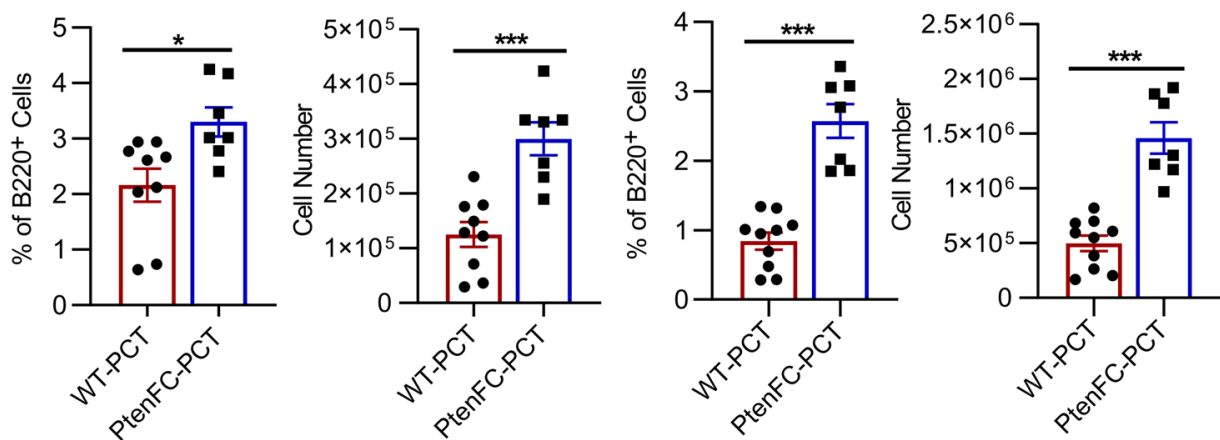

Figure 4. Augmented Tfr cell development promotes higher IgE and correlates with increased GC responses after food allergy sensitization. WT and PtenFC mice were sensitized with PCT as in Figure 1. On day 36 of the sensitization system, (A) serum was tested for peanut-specific Abs, and (B-D) SPs and mesenteric LNs were analyzed for Tfr, Tfh, and GCB cells by flow cytometry as in Figure 2. Representative contour dot plots for each cell staining are shown along with graphs indicating the average percentage of cells as a fraction of the parental cell population and the total yield of cells. Data for $\mathbf{A}$ are from 1 representative experiment of 2 experiments with 3-5 mice per group. Data for B-D were pooled from 2 experiments with 3-4 mice per group ( $n=$ 6-10). ${ }^{*} P<0.05,{ }^{* *} P<0.01$, and ${ }^{* * *} P<0.001$, by 2 -way ANOVA with Holm-Šidák multiple comparisons test (A) or 2-tailed Student's $t$ test (B-D). 
A

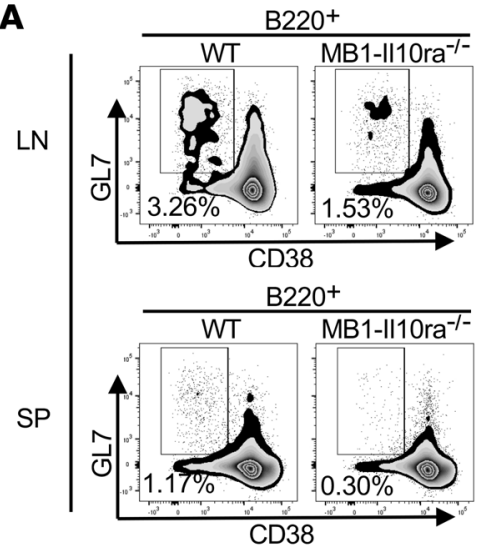

LNs - GCB \%
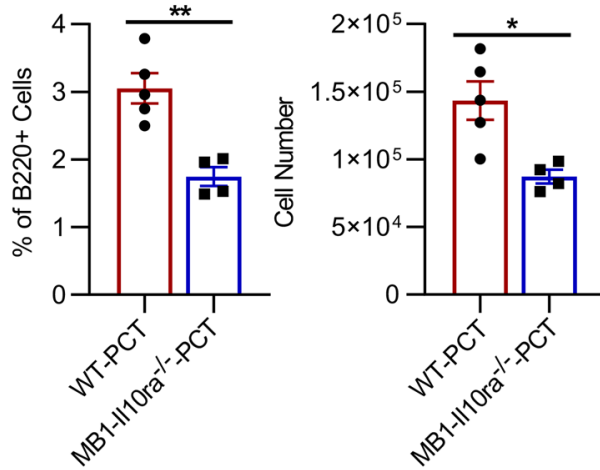

SPs - GCB \%

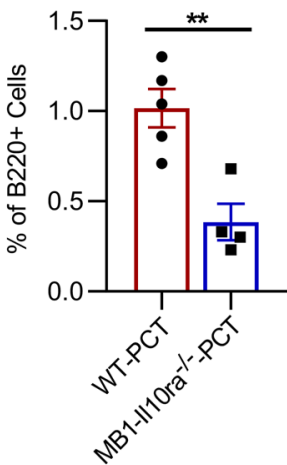

SPs - GCB nos.

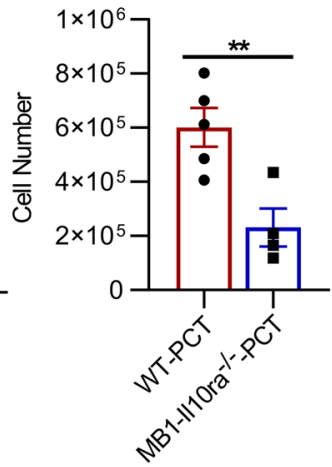

Total lgE
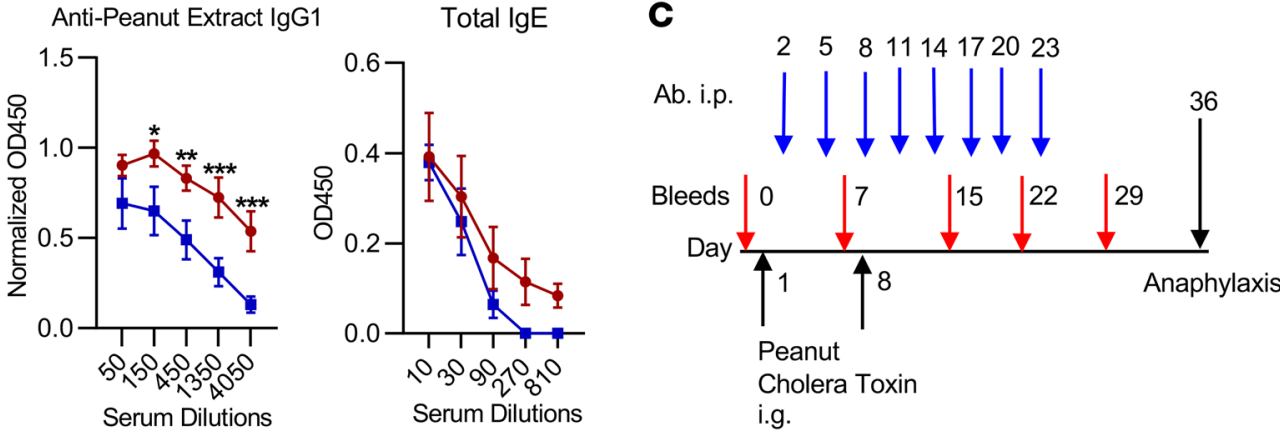

B Anti-Peanut Extract IgE

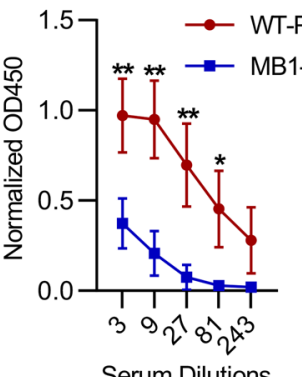

D Anti-Peanut Extract lgE

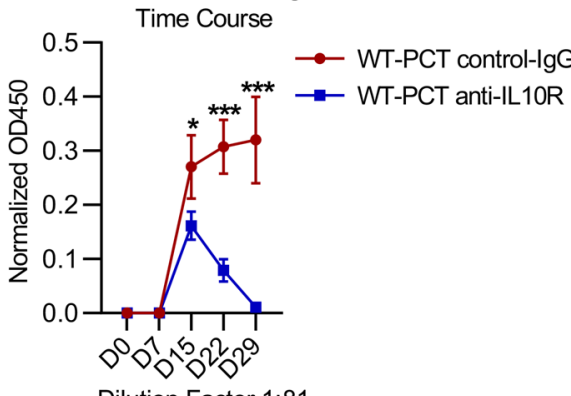

Dilution Factor 1:81

E Hypothermia

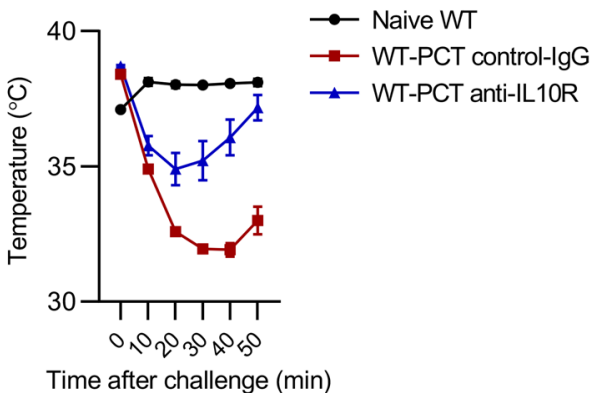

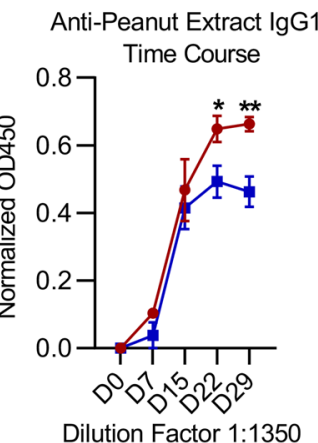

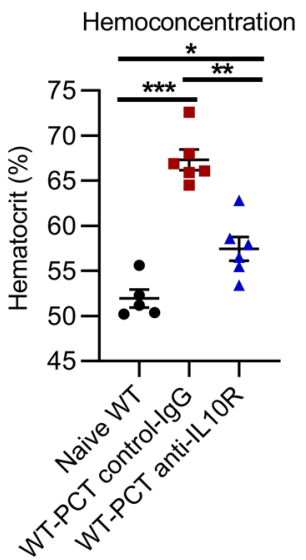

Clinical signs

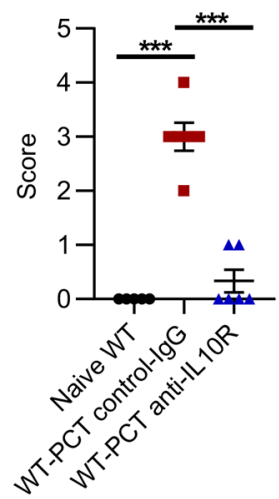

Figure 5. IL-10 promotes GCB cell levels and peanut-specific IgE, and therapeutic blockade of IL-10 during food allergy sensitization leads to loss of IgE. (A) WT and MB1-II10 ra-1- mice were sensitized with PCT. On day 36, GCB cells from LNs and SPs were stained and analyzed by flow cytometry, and GCB cells from LNs and SPs were stained and analyzed by flow cytometry. Representative contour dot plots of GCB cell staining are shown along with graphs indicating the average percentage of GCB cells and total yield of cells. (B) Peanut-specific IgE and IgG1 titers from day-36 serum of WT and MB1-II10ra ${ }^{-1-}$ mice sensitized with PCT. Data for $\mathbf{A}$ and B are from 1 representative experiment of 3 experiments with $3-5$ mice per group. (c) Schematic illustrating blockage of the IL-10R during PCT sensitization in female C57BL/6 WT mice. Numbers indicate the specific days for i.p. anti-IL-10R Ab treatment, i.g. PCT gavage, blood sampling, and anaphylaxis. Control mice received anti-HRP-IgG1 Ab. (D) Peanut-specific IgE and IgG1 titers from serum of control and anti-IL-10R mice treated as described in C at the indicated time points. (E) Anaphylaxis response of control and anti-IL-10R mice treated as described in C. Anaphylaxis analysis was performed as in Figure 1. Data for $\mathbf{D}$ and $\mathbf{E}$ are from 1 representative experiment of 2 experiments with 3-6 mice per group. ${ }^{*} P<0.05,{ }^{* *} P<0.01$, and ${ }^{* *} P$ $<0.001$, by 2-tailed Student's $t$ test (A) or 2-way ANOVA with Holm-Šidák multiple comparisons test (B and $\mathbf{D}$ ). 
A

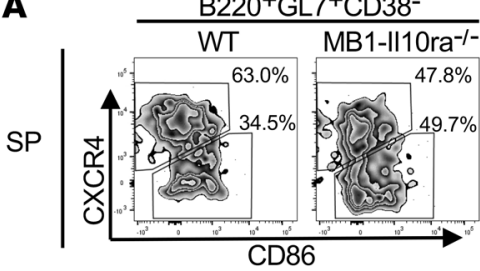

C

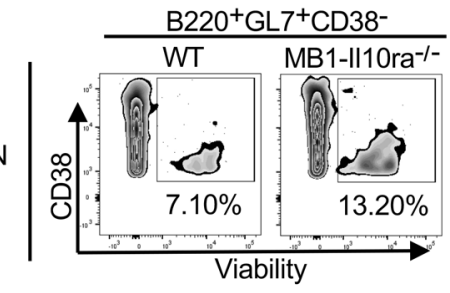

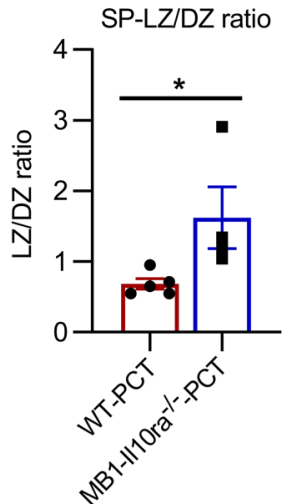

LN-Dead GCB\%

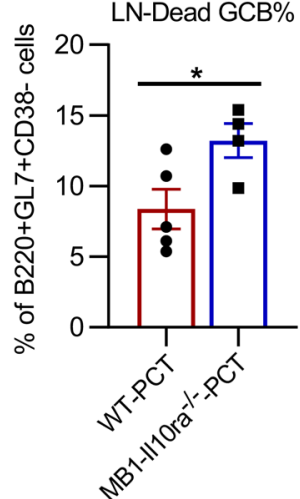

B

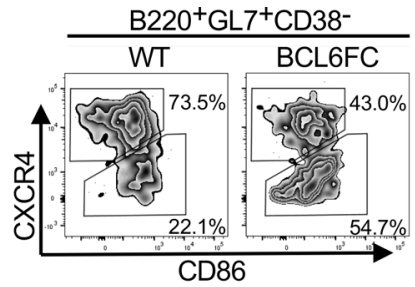

D

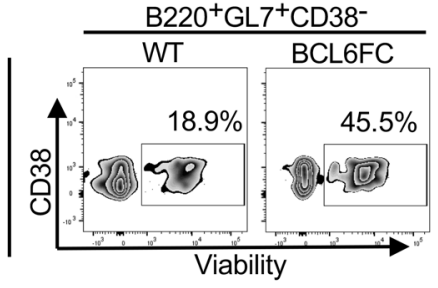

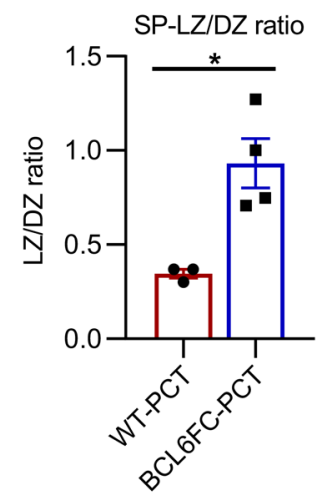

LN-Dead GCB\%

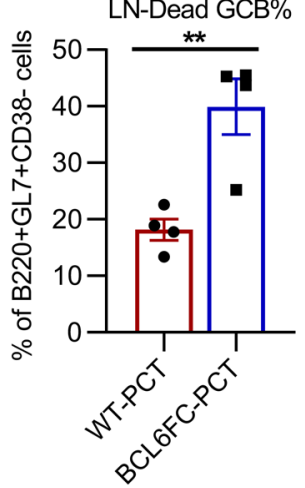

Figure 6. Altered GCB cell cycling and increased apoptosis in the absence of Tfr cells. (A) WT and MB1-II10ra- mice were sensitized with PCT. On day 36, GCB cells from SPs were stained and analyzed by flow cytometry for light zone (LZ) (CD86) and dark zone (DZ) (CXCR4) marker expression. Representative contour dot plots of GCB DZ/LZ cell staining are shown along with graphs indicating the average ratios of GCB LZ to GCB DZ cells. (B) WT and BcI6FC mice were sensitized with PCT. On day 36, GCB cells from SPs were stained and analyzed by flow cytometry for LZ and DZ marker expression as in A. Representative contour dot plots of GCB DZ/LZ cell staining are shown along with graphs indicating the average ratios of GCB LZ to GCB DZ cells. Data for $\mathbf{A}$ and $\mathbf{B}$ are from 1 representative experiment of 2 experiments with 4-5 mice per group. WT and Bcl6FC (C) and MB1-II10ra ${ }^{-/-}$(D) mice were sensitized with PCT. On day 36 CCB cells from LNs were stained and analyzed by flow cytometry for viability using eBioscience Fixable Viability Dye. Representative viability stains are shown along with graphs indicating the average percentage of GCB cell death. Data for $\mathbf{A}$ and $\mathbf{B}$ are from 1 representative experiment of 2 experiments with 4-5 mice per group. ${ }^{*} P<0.05$ and ${ }^{* *} P<0.01$, by 2-tailed Student's $t$ test $(\mathbf{A}-\mathbf{D})$.

GCB cell percentages all increased over time and remained high 4 weeks after the last sensitization, on day 36 (Figure 2C). The Tfh and GCB cell response was statistically normal at the early stages in Bcl6FC mice but was decreased at later stages of the analysis, with a sharp decrease in GCB cells observed on day 36 of the response. GCB cells from WT mice continued to expand up to day 36, but in Bcl6FC mice lacking Tfr cells, GCB cell growth leveled off by day 15 . The frequency of peanut-specific GCB cells was similar between WT and Bcl6FC mice (Supplemental Figure 7), indicating that the loss of peanut-specific IgE and IgG1 was due to the loss of absolute numbers of GCB cells and not to a loss in the frequency of peanut-specific B cells. The normal Tfh/GCB response at early time points is further evidence that the later decline in the Tfh/GCB response in Bcl6FC mice was not due to abnormal deletion of $\mathrm{Bcl} 6$ by FOXP3-Cre in Tfh cell precursors. We confirmed these trends in a more robust PCT priming model involving 8 PCT sensitization doses rather than our normal 2 PCT sensitization doses, showing that Tfr cells were still required even for a much stronger allergic induction (Supplemental Figure 8).

Tregs are required for Ag-specific IgE production in the food allergy model. We next wondered whether our results with Bcl6FC mice were unique to this mouse model of Tfr deficiency. To this end, we obtained Foxp3 diphtheria toxin receptor (FOXP3-DTR) mice, in which FOXP3 ${ }^{+}$Tregs could be deleted by injection of diphtheria toxin (DT), causing loss of Tfr cells $(10,36)$, and tested them in the PCT model, as shown in Figure 3A. Similar to our results with Bcl6FC mice, deletion of total Tregs led to a dramatic loss of peanut-specific IgE and a significant, but not complete, loss of peanut-specific IgG1 (Figure 3B). We then confirmed that the DT treatment led to thorough deletion of Tregs and Tfr cells at an early stage of the response in the FOXP3-DTR mice in both LNs and SPs (Figure 3, C and D, and Supplemental Figure 9A). These data support the idea that Tfr cells derived from FOXP3 ${ }^{+}$Tregs are actively required for producing Ag-specific IgE. Also, consistent with the results in Bcl6FC mice, we found that total IgE levels were strongly elevated after Treg deletion (Figure 3B). Unlike Bcl6FC mice, overall Tfh and GCB cell responses were increased after Treg deletion (Supplemental Figure 9, B and C), indicating that Tfh and GCB cell levels were repressed by Tregs and not by BCL6-dependent Tfr cells.

Tfr cells actively promote Ag-specific IgE. Although our experiments showed that Tfr cells were necessary for peanut-specific IgE responses in the PCT model, our data did not indicate whether Tfr cells were simply required at some minimal level to help the GC 

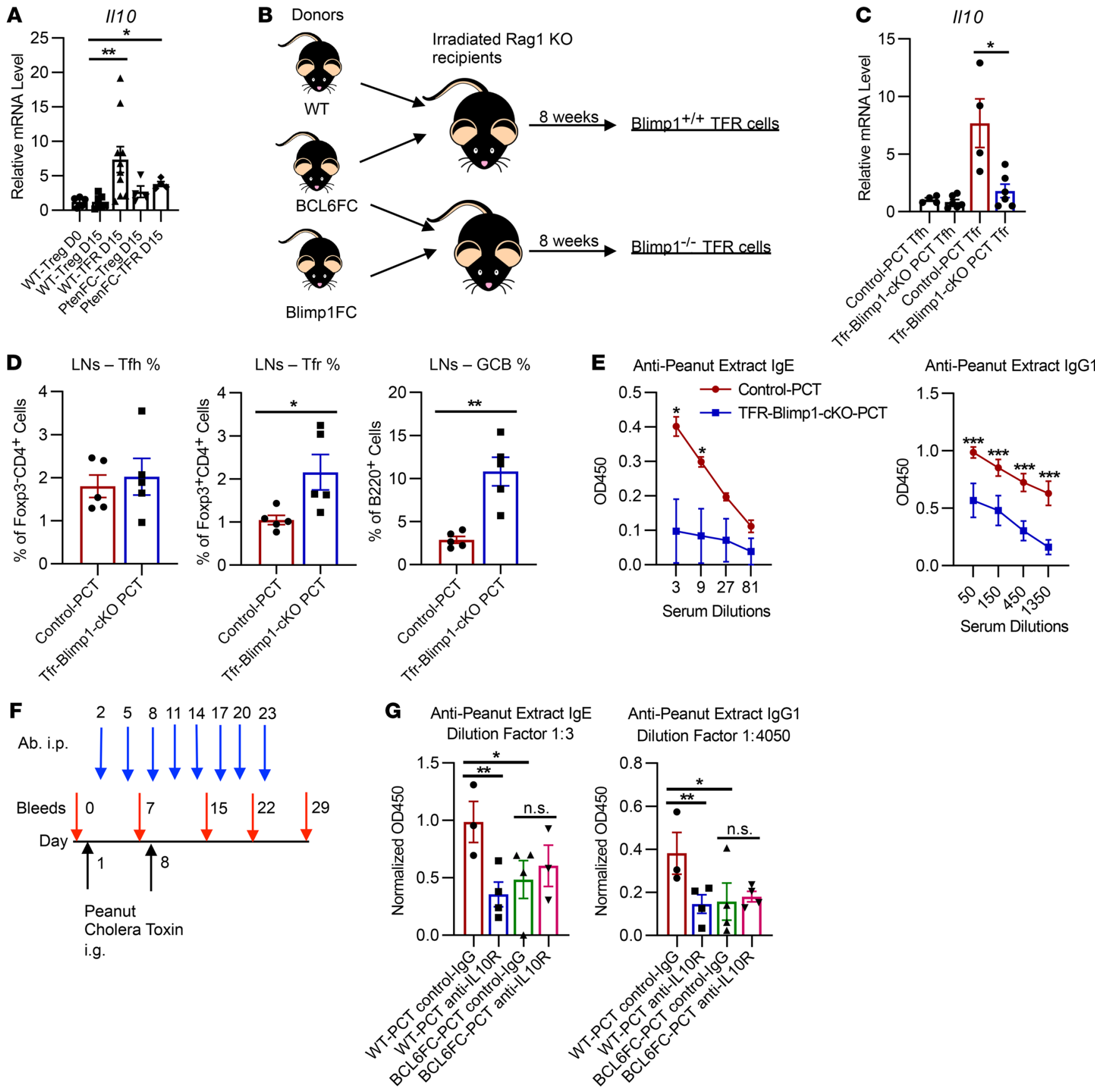

Figure 7. Blimp1-controlled Tfr cell-derived IL-10 is required for peanut-specific IgE production. (A) I/10 mRNA levels in Tregs or Tfr cells isolated from naive or PCT-sensitized WT mice on day 0 (naive) and day 15 as in the model shown in Figure 1. (B) Design for WT/Bcl6FC and Bcl6FC/Blimp1FC BM chimeras. (C-E) Mice generated as in B were sensitized with PCT. Then on day 36, (C) Tfh and Tfr cells were isolated by FACS, and I/10 expression was analyzed by qPCR; (D) Tfh, Tfr, and GCB cells from LNs were stained and analyzed by flow cytometry as in Figure 2; and (E) peanut-specific lgE and IgG1 titers from day-36 serum obtained from mice after PCT sensitization were measured. Data for A were pooled from 3 different cell sorts with 2-4 mice per sort $(n=6-10)$. Data for C-E are from 1 representative experiment of 2 experiments with 3-5 mice per group after PCT sensitization. (F) Scheme for blockade of IL-10R during PCT sensitization in WT and BcI6FC mice. Numbers indicate the specific days for i.p. anti-IL-10R Ab treatment, i.g. PCT gavage, blood sampling, and anaphylaxis. Control mice received anti-HRP-IgG1 Ab. (G) Peanut-specific IgE (day 15) and IgG1 (day 29) titers from serum obtained from control and anti-IL-10R-treated mice as described in $\mathbf{F}$. Data for $\mathbf{F}$ and $\mathbf{G}$ are from 1 representative experiment of 2 experiments with 3-4 mice per group. ${ }^{*} P<0.05$, ${ }^{* *} P<0.01$, and ${ }^{* * *} P<0.001$, by 2 -way ANOVA with Tukey's multiple comparisons test (A, C, and $\left.\mathbf{C}\right)$ or 2-tailed Student's $t$ test (D).

response or whether Tfr cells actively promoted peanut-specific GCB cell responses. We therefore used a mouse model previously shown to develop augmented Tfr responses due to a specific deletion of Pten expression in Tregs (Foxp3-Cre Pten ${ }^{f / f l}$, referred to here- after as PtenFC mice; ref. 37) to test this idea. We sensitized PtenFC mice with PCT and analyzed the resulting immune response. As shown in Figure 4A, peanut-specific IgE and IgG1 were strongly increased in PtenFC mice compared with levels in WT mice. 
We confirmed that Tfr cells were significantly augmented in the PCT-sensitized PtenFC mice (Figure 4B) and observed that this increase was associated with an increase in both Tfh and GCB cells (Figure 4, C and D). These data directly challenge the notion that Tfr cells act as suppressors of the GC response, as is standard dogma for Tfr function. Instead, our data showed that Tfr cells actively drove GC responses in this food allergy model. This idea is supported by a linear correlation analysis between the numbers of Tfr cells and other cells in the GC (Supplemental Figure 10A). As expected, the numbers of Tfh and GCB cells were tightly correlated, but Tfr cells also positively correlated with both GCB and Tfh cells to a highly significant degree $(P<0.0001)$. This finding, coupled with the diminished GCB cell response in Bcl6FC mice, indicates that Tfr cells acted as true helper cells of the Ab response in the PCT food allergy model. We did not observe abnormal expression of Il4, Il21, or Ifng by Tfh cells or Tfr cells from PtenFC mice (Supplemental Figure 10B), suggesting that the results we observed following PCT challenge in these mice were not due to aberrant expression of those key Tfh cytokines.

$I L-10$ is critical for Ag-specific IgE responses. Tfr cells produce IL-10 that can augment the GC and Ab response (16). We wondered whether Tfr cell-derived IL-10 was playing a similar helper role in our food allergy model. Since Bcl6FC mice lack Tfr cells, the smaller GC responses in Bcl6FC mice are consistent with loss of Tfr cell-derived IL-10 acting on GCB cells. To specifically test the role of IL-10 in regulating peanut-specific IgE in our food allergy model, we used Mb1-Cre IL-10Ra $a^{f / f l}\left(\mathrm{MB1}-\mathrm{Il1Ora}^{-/}\right)$mice, in which the IL-10 receptor $\alpha$ gene is deleted specifically in B cells and the $\mathrm{B}$ cells cannot respond to IL-10 signals. MB1-Il10ra- ${ }^{-/}$mice were sensitized with PCT and tested for GC and peanut-specific $\mathrm{Ab}$ responses. As shown in Figure 5, A and B, loss of IL-10 signaling in B cells led to significantly decreased GCB cell, peanut-specific IgG1, and peanut-specific IgE responses. Baseline Tfh, Tfr, and GCB levels were normal in MB1-Il10 $\mathrm{ra}^{-/-}$mice, indicating no broad immune defect from loss of IL-10 signaling in these mice (Supplemental Figure 11, A-C). Previously, neutralizing IL-10 Abs were shown to decrease IgE and gastrointestinal symptoms in an oral challenge model (38). We then wondered whether we could block IgE production with anti-IL-10R Ab in our peanut model. As shown in Figure 5, C and D, repeated doses of anti-IL-10R Ab in WT mice after PCT challenge led to a dramatic loss of peanut-specific IgE by day 29, with a significant drop in peanut-specific IgG1 by day 29. Importantly, anti-IL-10R Ab treatment also strongly inhibited anaphylaxis after peanut challenge (Figure 5E), mirroring the loss of peanut-specific IgE. We tested the role of IL-10 signaling on CD4 ${ }^{+}$ T cells with Cd4-Cre Il10rafl/fl mice and the PCT sensitization system. Although we noted a slight decrease in Tfh cell development in these mice, there was no difference in anti-peanut IgE and IgG1 or GCB cell responses (Supplemental Figure 11, D-G). These data support the idea that IL-10 acts directly on GCB cells and not on Tfh cells to promote the IgE response. We next examined the light zone and dark zone composition of GCB cells in MB1-Il10 ra $^{-/-}$mice and found a large shift to light zone GCB cells in these mice (Figure $6 \mathrm{~A})$. These data are consistent with recent findings that IL-10 promotes entry of the GCB cell into the dark zone compartment of the GC (16) and that without IL-10 signaling, GCB cells accumulate in the light zone. We also observed a shift of GCB cells to the light zone in Bcl6FC mice (Figure 6B), consistent with a loss of Tfr cell-derived IL-10 acting on GCB cells. Furthermore, we observed a significant increase in apoptotic GCB cells in both MB1-Il10ra ${ }^{-/-}$ and Bcl6FC mice (Figure 6, C and D), which helps explain the loss of GCB cells in both strains of mice.

Tfr cell-derived IL-10 promotes Ag-specific IgE responses. To further link Tfr cell-derived IL-10 to peanut-specific IgE, we first examined Il10 mRNA expression from FACS-isolated Tfr cells after PCT sensitization (Figure 7A). On day 15 after sensitization, we observed a significant increase in $I l 1 O$ produced by WT Tfr cells compared with WT Tregs, which correlated with the appearance of IgE in the serum. Tfr cells from PtenFC mice produced less Il1O mRNA than did control Tfr cells, however, the difference was not statistically significant (Figure 7A). We next conducted experiments to link IL-10 made by Tfr cells directly to IgE production. First, we used a bone marrow (BM) chimera approach, in which we introduced either WT plus Bcl6FC BM or Blimp1 $1^{f / f l}$ Foxp3-Cre (Blimp1FC) plus Bcl6FC BM into irradiated Rag1 ${ }^{-/}$mice (Figure 7B). Blimp1 is essential for IL-10 transcription in Tregs $(39,40)$. Gene profiling of Tfr cells from WT and Blimp1FC mice showed that the major Treg and Tfr suppressor genes, with the exception of Il10, were expressed normally in Blimp1FC cells (Supplemental Figure 12), and a decrease in Il1O transcripts in Tfr cells from Blimp1FC plus Bcl6FC chimeras was confirmed by quantitative PCR (qPCR) of FACS-isolated Tfr cells (Figure 7C). The data in Figure $7 \mathrm{C}$ also show that Tfr cells expressed at least 7 times more $I l 10$ than did Tfh cells. Chimeras with WT plus Bcl6FC BM will develop WT Tfr cells (Figure 7B), and after PCT sensitization of these mice, we observed substantial levels of Tfr, Tfh, and GCB cells in LNs from these mice (Figure 7D), as well as a robust peanut-specific IgE and IgG1 response (Figure 7E). Chimeras with Blimp1FC plus Bcl6FC BM will only develop Blimp1 $1^{-/-}$Tfr cells (Figure 7B), and after PCT sensitization, these mice had augmented Tfr cell development, as expected (41), as well as a significant increase in GCB cells (Figure 7D). Strikingly, Blimp1FC plus Bcl6FC BM chimeras had severely attenuated peanut-specific IgE and IgG1 responses (Figure 7E). These data show that Tfr cells incapable of producing normal IL-10 levels cannot drive the Ag-specific IgE response. Additionally, we found that anti-IL-10R Ab inhibited the peanut-specific IgE response in WT control mice to the level seen in Bcl6FC mice, whereas anti-IL-10R Ab did not inhibit the IgE response in Bcl6FC mice (Figure 7, F and G, and Supplemental Figure 13). These 2 lines of evidence indicate that production of IL-10 by Tfr cells was required for peanut-specific IgE production.

\section{Discussion}

Here, we show that Ag-specific IgE responses induced in a food allergy sensitization system were strikingly dependent on Tfr cells and, further, that Tfr cells played an active helper role in the production of Ag-specific IgE. Our data also point to a key role of IL-10 produced by Tfr cells in promoting the development of Ag-specific IgE-secreting cells from the GC. The data presented here fit into an emerging helper model of Tfr cell function that both refines and revises the standard view of Tfr cells.

Initial studies of Tfr cells described them as suppressors of the $\mathrm{GC}$ and $\mathrm{Ab}$ response, however, more recent studies have indicated that Tfr cells can have a helper function. Our initial work 
with Bcl6FC mice showed an unexpected loss of Ag-specific IgG after immunization (13). Laidlaw et al. analyzed Tfr function in a virus infection model and showed that Tfr cells produce IL-10 within the GC to facilitate GCB cell growth and Ag-specific IgG production (16). Here, in a food allergy model, we show a strong positive regulation of Ag-specific IgE by Tfr cells and IL-10 and, further, that Tfr cells were critically required for the allergic IgE response to develop.

Strikingly, the augmented Tfr cells in PtenFC mice drove dramatically higher levels of peanut-specific IgE compared with that seen in WT mice, strongly supporting a positive helper role for Tfr cells in this model, in contrast to the common view that Tfr cells act as suppressors of the GC response $(9-11,14,15)$. We further note that Pten-deficient Tfr cells strongly promoted a Th2-type IgE response despite being linked previously to aberrant Th1-type inflammatory disease (37). Indeed, in our model, Tfh and Tfr cells from PtenFC mice did not overproduce IFN- $\gamma$ (Supplemental Figure 10), as was previously reported (37). These data suggest that Tfr and Tfh cell cytokine expression can be influenced by the type of immune response.

Relatively little work has been done on the regulation of IgE by Tfr cells. In 2014, Wing et al. published data that Treg deletion and specific deletion of Ctla4 in Tregs led to a large increase in NP-specific IgE after NP-OVA-alum immunization (36). One interpretation of these studies is that Tfr cells, which were affected in these model systems, strongly suppress IgE. However, since these experiments globally affected Tregs, a different and equally feasible explanation is that the IgE responses were enhanced by the loss of Tregs or Treg-expressed CTLA4 at the early stages of the immune responses, leading to greater inflammation, stronger Tfh and GCB cell responses, and subsequent higher production of Ag-specific IgE. Thus, we propose that an amplified early Tfh cell expansion effectively overshadows the subtler effects of Tfr cells on the GC response. More recently, using an alternative strategy to delete Tfr cells in "Tfr-DTR" mice, Clement et al. showed that Tfr cells could suppress Ag-specific IgE in a house dust mite-induced (HDM-induced) airway inflammation model (42). This result suggests that either Tfr cells regulate Ag-specific IgE differently after airway challenge than after gut challenge, or that in the Tfr-DTR mouse system, global Treg responses are also affected, leading to greater inflammation from defective early Treg-mediated suppression. In support of this latter interpretation, overall lung inflammation was significantly increased in the Tfr-DTR mice (42).

Although some groups have shown that Tfr cells function as strong suppressors of B cell responses using in vitro culture systems, such cultures are unlikely to recapitulate the highly organized structure of the GC in vivo and thus may not reflect normal Tfr functions (15). Studies of Tfr cells in vivo are therefore key to understanding Tfr cell functions. Since mouse models are typically used to study in vivo Tfr cell function, little is known about human Tfr cell function in vivo. Furthermore, there is controversy about how to define human Tfr cells within lymphoid tissue. Although CD4 ${ }^{+}$ $\mathrm{CXCR}^{+} \mathrm{PD}-1^{++} \mathrm{BCL}^{+} \mathrm{FOXP}^{+} \mathrm{Tfr}$ cells are readily detected in mouse lymphoid tissues after immunization, analogous cell populations have been hard to find in human lymphoid tissues that contain GCs (43-45). Whether tissue-resident human Tfr cells uniformly express FOXP3, PD-1, or CD25 remains to be clarified (43-45).
Human Tfr-like cells defined as $\mathrm{CD} 4^{+} \mathrm{CXCR} 5^{+} \mathrm{PD}-1^{++} \mathrm{CD} 25^{+} \mathrm{FOXP} 3^{-}$ can suppress $\mathrm{B}$ cell and IgE responses in vitro via IL-10 secretion (43), but as noted above, this may not reflect the in vivo setting. Whether there are very rare Tfr cells in humans that are clear counterparts to mouse Tfr cells or whether human Tfr cells consist of a variety of related follicular $\mathrm{T}$ cell populations that are distinct from mouse Tfr cells remains to be determined. Circulating Tfr cells have been examined extensively in humans $(35,43,46-48)$, however, the relationship of these cells to tissue-resident Tfr cells is not known. Much work remains to be done on human Tfr cells.

One question that emerges from our study is why Ag-specific IgE responses appear to be much more sensitive to the loss of $\mathrm{Tfr}$ cells than Ag-specific IgG responses. The answer may reflect the unique nature of IgE as an Ag receptor for B cells in the GC. Specifically, $\mathrm{IgE}^{+}$switched GCB cells have altered Ag receptor signaling, differentiate more readily into plasmablasts, display increased apoptosis, and are less efficiently selected than are GCB cells expressing other Ig isotypes $(25,27,28,49-51)$. Furthermore, inhibition of apoptosis in the GC helps to promote IgE responses (52). We hypothesize that Tfr-derived IL-10 is essential to maintain the survival and clonal expansion of $\operatorname{IgE}^{+} \mathrm{GCB}$ cells by promoting entry into the GC dark zone, where they can proliferate rather than differentiate or undergo apoptosis.

The strong decrease in Tfh cells in Bcl6FC mice probably relates to the role of $\mathrm{Tfr}$ cells in maintaining the $\mathrm{GC}$ reaction via IL-10. We show here that IL-10 from Tfr cells promoted GCB cell survival, and in the absence of this IL-10, we observed fewer GCB cells. Since Tfh cells depend on GCB cells for stimulation, Tfh cell levels necessarily correlate with GCB cell levels, so that fewer GCB cells leads to fewer Tfh cells $(53,54)$. Recently, a Tfh cell subset, termed TFH13 for their expression of IL-13, was shown to drive the generation of high-affinity IgE Abs (55). To test whether Tfr cells affect the development of TFH13 cells, we isolated Tfh cells from WT and Bcl6FC PCT-sensitized mice and analyzed cytokine expression by qPCR (Supplemental Figure 14). We found no clear difference in the expression of Il4 or Il13 between WT and Bcl6FC Tfh cells, suggesting that defects in Tfh 13 cell development did not explain the loss of IgE in Bcl6FC mice.

One interesting question is why we observed larger GCs in our Bcl6FC plus Blimp1FC BM chimeras, if Blimp1FC-deficient Tfr cells are deficient in IL-10 production. In other words, if Tfr cell-derived IL-10 is critical for GC size, then we should have seen fewer GCB cells in the chimeras. There are 3 potential explanations for our results of larger GCs in the Bcl6FC plus Blimp1FC chimera. First, the Tregs in the chimeras produced less IL-10 on average than did Tregs from control mice. Thus, background immune responses may have been amplified from lower levels of IL-10, leading to larger overall GCs. Second, lower levels of IL-10 on average from Tregs amplified early $\mathrm{T}$ cell activation after PCT sensitization, leading to overall larger GCs. Third, loss of Blimp1 in Tregs led to a lack of repression of Bcl6, and thus Tfr cell differentiation occurred at a higher rate, as shown in Figure 7D. We propose that Tfr cells have additional B cell helper activity from factors besides IL-10 that is manifested in these chimeras due to the higher rate of Tfr cell development. Indeed, as shown in Supplemental Figure 12, Tfr cells produced Il4, Il21, and Tnfsf13b (also known as B cell-activating factor [BAFF]), cytokines which can all 
help promote $\mathrm{B}$ cell responses. We did not observe an increased Tfh cell response in the chimeras (Figure 7D), indicating that there was no general increase in immune reactions. These data support the third explanation that Tfr cells have additional GCB cell helper activity besides that from IL-10. We should also note that, recently, Wang et al. published a study indicating that Blimp1-deficient Tfr cells are unstable and can differentiate into IL-17-producing cells that can produce IL-4 and IL-21 and promote larger GCs (56). Although this model could explain the discrepancy of larger GCs despite depleted IL-10 production from the Tfr cells, we found no evidence in our RNA-Seq data of higher Il17 in Blimp1-deficient Tfr cells (Supplemental Figure 12). This discrepancy could be due to the gut immune challenge model we used versus the OVA-Alum i.p. immunization the authors used, as well as other factors such as the microbiome, mouse housing, and genetic variations.

We also note that our study shows that Tfr cells were also required to repress non-peanut-specific IgE responses. We hypothesize that the accumulation of large numbers of nonspecific IgE GCB cells in Tfr-deficient responses leads to consumption of the available IL-10 in the GC and deprives the peanut-specific IgE GCB cells of critical IL-10, thus weakening the peanut-specific IgE GCB cell response. Another explanation for the role of Tfr-derived IL-10 in the Ag-specific IgE response is that GCs induced in Th2-type responses are inherently weaker than GCs induced in Th1-type responses because of a lack of strong inflammatory signals (57) and are more dependent on IL-10 from Tfr cells to maintain the response.

IL-10 has recently become recognized as a positive factor in $\mathrm{Ab}$ responses and particularly GCB cell responses (16, 58-60), and our study shows the positive regulation of food $\mathrm{Ag} \operatorname{IgE}$ responses by IL-10. Since IL-10 is a positive mediator of IgE responses, this finding could be exploited to inhibit IgE-mediated allergy. We show that blocking IL-10 signaling after initiation of the peanut allergy sensitization can potently inhibit the production of peanut-specific IgE. These data have significant implications for therapies designed to treat food allergies and other IgE-mediated allergic diseases. Importantly, IL-10 is also a key cytokine involved in the suppression of immune responses and is part of the mechanism of allergen-specific immunotherapy (61-67). IL-10 produced by Tregs, Bregs, Tr1 cells, and other immune cell subsets has been shown to play an important role in suppressing undesired immune responses (61-67). Thus, there is an apparent dichotomy in the role of IL-10 in allergic regulation. Our hypothesis is that IL-10 acts as a general immune-suppressive factor at the early stages of $\mathrm{T}$ cell activation in the allergic immune response, but once GC reactions are formed, IL-10 has a unique role in promoting the GC and IgE-expressing B cells. Clearly, any therapy that involves manipulation of IL-10 signaling must take these dual roles into account. We observed that blocking IL-10 after immunization could block the development of peanut-specific IgE responses. Further work on the timing of in vivo IL-10 blockade in the development of food allergy will be important. Although IL-10 typically acts as an immune-suppressive factor when tested in vitro, IL-10 functions are also known to be pleiotropic (68), and recent work including the present study supports the idea of IL-10 pleiotropy in vivo $(16,58)$.

Last, we note that patients with immune dysregulation, polyendocrinopathy, enteropathy, X-linked (IPEX) syndrome often have elevated IgE levels and food allergy (69-71). These patients have mutations in the FOXP3 gene and thus defective Treg and Tfr cell function. The elevation of IgE in these patients fits with our results, in which disruption of Tfr cells or Treg function led to strongly increased total serum IgE. How patients with IPEX syndrome develop food allergy or food Ag-specific $\operatorname{IgE}$ is not known, but the severe immune dysregulation in this syndrome appears to be able to overcome the loss of Tfr cells for the production of $\operatorname{IgE}$ that is reactive with food Ags. Further research is required to understand the development of food allergy-promoting IgE in humans.

Overall, our study increases our understanding of the regulation of IgE responses and elucidates the complex function of $\mathrm{Tfr}$ cells in the GC.

\section{Methods}

Mice. All mutant mice were on a C57BL/6 background. Foxp $3^{\text {YFP-Cre }}$ (WT), Foxp3 $3^{\text {YFP-Cre }} \mathrm{Bcl}^{f / / f l}(\mathrm{Bcl} 6 \mathrm{FC}), B c l 6^{f / f l}$, and Cd4-Cre Bcl6 $6^{f / f l}$ (CD4-BCL6-cKO) mice were described previously (12, 34). B6.129

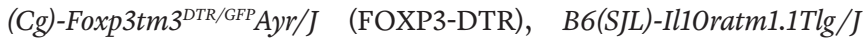
(Il1Ora $\left.{ }^{\text {fl/fl }}\right)$, B6.129S7-Rag1tm1Mom/J (Rag1-/-), B6;129P2-Fcerlg ${ }^{\text {tmlRav } / J}$ $\left(F c \varepsilon R 1^{-1}\right)$, and WT C57BL/6J mice were obtained from The Jack-

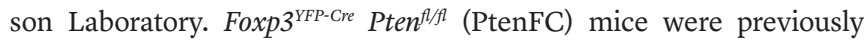
described (37). $\mathrm{Mb1}$-Cre Bcl6 $6^{f / f l}$ (MB1-Bcl6 ${ }^{--}$) mice were obtained from Marion Pepper (University of Washington, Seattle, Washington, USA).

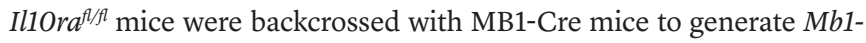
Cre Il1Ora ${ }^{f / f l}\left(\mathrm{MB1}-\mathrm{Il1Ora}^{-/}\right)$mice. Il1Ora ${ }^{\mathrm{f} / \mathrm{fl}}$ mice were also backcrossed with $\mathrm{Cd} 4$-Cre mice to generate $\mathrm{Cd} 4$-Cre Il1Orat/fll $\left(\mathrm{CD} 4-\mathrm{Il1Ora}^{-/ /}\right)$mice. For Bcl6FC or PtenFC mice, only FOXP3-YFP-Cre mice were used as WT controls. For MB1-Il10ra ${ }^{-/-}$or CD4-Il10ra ${ }^{-/-}$mice, only Il1Orat/fl mice were used as controls. Only Bcl6 $6^{f / f l}$ mice were used as controls for CD4-BCL6-cKO mice. All mice, including conditional knockout mice, were on a C57BL/6 background. Six- to 10-week-old male and female mice were used for most experiments. For anaphylaxis assays, female mice were used, as they produced stronger responses. Mouse littermate comparisons were used whenever possible. Control and experimental mouse cohorts were age and sex matched. Mice were bred under specific pathogen-free conditions at the laboratory animal facility of the Indiana University School of Medicine.

Peanut sensitization. On the indicated dates, the mice were starved for 2 hours, and then each mouse was fed $300 \mu \mathrm{L} 1.5 \% \mathrm{NaHCO}_{3}$ water (i.g). A half hour later, each mouse was sensitized with $1 \mathrm{mg}$ peanut extract (Greer Laboratories) or OVA (MilliporeSigma) together with 10 $\mu \mathrm{g}$ cholera toxin (MilliporeSigma) (31-33). The mice were sacrificed on the indicated days, serum was collected, and the mesenteric LNs and SPs were harvested. For serum time course analyses, approximately $0.3 \mathrm{~mL}$ blood was collected from each mouse from the submandibular vein on the indicated dates.

Assessment of anaphylaxis. To assess anaphylaxis, $2 \mathrm{mg}$ peanut extract protein without cholera toxin was administered i.p. per mouse 4 weeks after the second immunization (day 36), similar to previous studies (31-33). Mice were monitored for 50 minutes after challenge for a change in rectal (core) body temperature (Braintree Scientific). After 50 minutes, whole blood was collected into EDTA-coated tubes (BD Microtainer), and hematocrit values were determined with an Element HT5 Veterinary Hematology Analyzer (Heska). Clinical scores were evaluated 20-30 minutes after challenge as reported previously (31-33). Briefly the animals were scored as follows: 0 , no clin- 
ical signs; 1 , scratching around the head and nose; 2 , reduced activity with increased respiration; 3 , wheezing and lying prone; 4 , no response after prodding and convulsion; and 5, death.

Additional methods and a list of the Ab clones used are provided in the Supplemental Methods.

Statistics. All data analysis was performed using GraphPad Prism software (GraphPad Software). Graphs show the mean \pm SEM. Unless otherwise stated, a 2-tailed Student's $t$ test or 2-way ANOVA with Tukey's post hoc analysis was used. All ELISAs were analyzed using 2-way ANOVA with Holm-Šidák multiple comparisons test. Only significant differences $(P<0.05)$ are indicated in the Figures. The investigators were not blinded for the analyses.

Study approval. All experiments and handling of animals were conducted according to protocols approved by the IACUC of the Indiana University School of Medicine.

\section{Author contributions}

MMX performed the experiments and generated most of the data shown, with experimental assistance from QC, BK, and HW. HL assisted with mouse breeding and genotyping. KY provided the PtenFC mice. SJM and BKH provided Ara $\mathrm{H} 1$ and Ara $\mathrm{H} 3$ peanut proteins. JCM provided the FceR1-KO mice. MHK gave general advice and helped design the $\operatorname{IgE}$ affinity experiment.
ALD and MMX jointly planned and conceived of the experiments and cowrote the manuscript.

\section{Acknowledgments}

We would like to thank Caleb Corn for help with mouse genotyping and Baohua Zhou and Charlie X Dong for critical evaluation of the data. We thank Tony Sinn for assistance with mouse irradiation and BM transplantation. We thank Marion Pepper for guidance with the B cell tetramer protocol. We would also like to thank Gowthaman Uthaman and Stephanie Eisenbarth for sharing their antibody affinity measurement protocol. This work was supported by NIH grants R01 AI132771 (to ALD) and R01 AI057459 and R01 AI129241 (to MHK). MMX was supported by a Careers in Immunology Fellowship from the American Association of Immunologists. Core facility usage was also supported by Indiana University Simon Cancer Center Support grants P30 CA082709 and U54 DK106846. Support provided by the Herman B Wells Center was in part from the Riley Children's Foundation.

Address correspondence to: Alexander L. Dent, 950 West Walnut Street, R2 302, Indiana University School of Medicine, Indianapolis, Indiana 46202, USA. Phone: 317.274.7524; Email: adent2@iupui.edu.
1. Victora GD, Nussenzweig MC. Germinal centers. Annu Rev Immunol. 2012;30:429-457.

2. MacLennan IC. Germinal centers. Annu Rev Immunol. 1994;12:117-139.

3. Klein U, Dalla-Favera R. Germinal centres: role in B-cell physiology and malignancy. Nat Rev Immunol. 2008;8(1):22-33.

4. Crotty S, Johnston RJ, Schoenberger SP. Effectors and memories: Bcl- 6 and Blimp- 1 in T and B lymphocyte differentiation. Nat Immunol. 2010;11(2):114-120.

5. McHeyzer-Williams LJ, Pelletier N, Mark L, Fazilleau N, McHeyzer-Williams MG. Follicular helper T cells as cognate regulators of B cell immunity. Curr Opin Immunol. 2009;21(3):266-273.

6. Vinuesa CG, et al. A RING-type ubiquitin ligase family member required to repress follicular helper T cells and autoimmunity. Nature. 2005;435(7041):452-458.

7. Linterman MA, et al. Follicular helper T cells are required for systemic autoimmunity. J Exp Med. 2009;206(3):561-576.

8. Odegard JM, et al. ICOS-dependent extrafollicular helper $\mathrm{T}$ cells elicit IgG production via IL-21 in systemic autoimmunity. J Exp Med. 2008;205(12):2873-2886

9. Wollenberg I, et al. Regulation of the germinal center reaction by Foxp ${ }^{+}$follicular regulatory $\mathrm{T}$ cells. J Immunol. 2011;187(9):4553-4560.

10. Linterman MA, et al. Foxp $3^{+}$follicular regulatory $\mathrm{T}$ cells control the germinal center response. Nat Med. 2011;17(8):975-982.

11. Chung Y, et al. Follicular regulatory T cells expressing Foxp3 and Bcl-6 suppress germinal center reactions. Nat Med. 2011;17(8):983-988.

12. $\mathrm{Wu} \mathrm{H}$, et al. Follicular regulatory $\mathrm{T}$ cells repress cytokine production by follicular helper $\mathrm{T}$ cells and optimize IgG responses in mice. Eur J Immunol. 2016;46(5):1152-1161.
13. Wu H, Xie MM, Liu H, Dent AL. Stat3 Is Important for Follicular Regulatory T Cell Differentiation. PLoS ONE. 2016;11(5):e0155040.

14. Sage PT, Sharpe AH. T follicular regulatory cells. Immunol Rev. 2016;271(1):246-259.

15. Xie MM, Dent AL. Unexpected help: follicular regulatory T cells in the germinal center. Front Immunol. 2018;9:1536.

16. Laidlaw BJ, et al. Interleukin-10 from CD4 follicular regulatory $\mathrm{T}$ cells promotes the germinal center response. Sci Immunol. 2017;2(16):eaan4767.

17. Botta D, et al. Dynamic regulation of T follicular regulatory cell responses by interleukin 2 during influenza infection. Nat Immunol. 2017;18(11):1249-1260.

18. Fu W, et al. Deficiency in T follicular regulatory cells promotes autoimmunity. J Exp Med. 2018;215(3):815-825.

19. Xie MM, Fang S, Chen Q, Liu H, Wan J, Dent AL. Follicular regulatory $\mathrm{T}$ cells inhibit the development of granzyme B-expressing follicular helper T cells. JCI Insight. 2019;4(16):128076.

20. Sicherer SH, Sampson HA. Peanut allergy: emerging concepts and approaches for an apparent epidemic. J Allergy Clin Immunol. 2007;120(3):491-503.

21. Asher MI, et al. Worldwide time trends in the prevalence of symptoms of asthma, allergic rhinoconjunctivitis, and eczema in childhood: ISAAC Phases One and Three repeat multicountry cross-sectional surveys. Lancet. 2006;368(9537):733-743.

22. Dullaers M, De Bruyne R, Ramadani F, Gould HJ, Gevaert P, Lambrecht BN. The who, where, and when of IgE in allergic airway disease. J Allergy Clin Immunol. 2012;129(3):635-645.

23. Gould HJ, et al. The biology of IGE and the basis of allergic disease. Annu Rev Immunol.
2003;21:579-628.

24. Talay $\mathrm{O}$, et al. $\operatorname{IgE}^{+}$memory B cells and plasma cells generated through a germinal-center pathway. Nat Immunol. 2012;13(4):396-404.

25 . He JS, et al. The distinctive germinal center phase of $\operatorname{IgE}^{+} \mathrm{B}$ lymphocytes limits their contribution to the classical memory response. J Exp Med. 2013;210(12):2755-2771.

26. Xiong H, Dolpady J, Wabl M, Curotto de Lafaille MA, Lafaille JJ. Sequential class switching is required for the generation of high affinity IgE antibodies. JExp Med. 2012;209(2):353-364.

27. Yang Z, Sullivan BM, Allen CD. Fluorescent in vivo detection reveals that $\operatorname{IgE}(+) \mathrm{B}$ cells are restrained by an intrinsic cell fate predisposition. Immunity. 2012;36(5):857-872.

28. Yang Z, Robinson MJ, Allen CD. Regulatory constraints in the generation and differentiation of IgE-expressing B cells. Curr Opin Immunol. 2014;28:64-70.

29. Kobayashi T, Iijima K, Dent AL, Kita H. Follicular helper T cells mediate IgE antibody response to airborne allergens. J Allergy Clin Immunol. 2017;139(1):300-313.e7.

30. Meli AP, Fontés G, Leung Soo C, King IL. T follicular helper cell-derived IL-4 is required for IgE production during intestinal helminth infection. J Immunol. 2017;199(1):244-252.

31. Li XM, et al. A murine model of peanut anaphylaxis: $\mathrm{T}$ - and $\mathrm{B}$-cell responses to a major peanut allergen mimic human responses. J Allergy Clin Immunol. 2000;106(1 Pt 1):150-158.

32. Chu DK, et al. IL-33, but not thymic stromal lymphopoietin or IL-25, is central to mite and peanut allergic sensitization. J Allergy Clin Immunol. 2013;131(1):187-200.e1.

33. Jiménez-Saiz R, et al. Lifelong memory responses perpetuate humoral $\mathrm{T}_{\mathrm{H}} 2$ immunity and anaphylaxis in food allergy. J Allergy Clin Immunol. 
2017;140(6):1604-1615.e5.

34. Hollister K, et al. Insights into the role of Bcl6 in follicular Th cells using a new conditional mutant mouse model. J Immunol. 2013;191(7):3705-3711.

35. Schulten V, et al. Allergen-specific immunotherapy modulates the balance of circulating Tfh and Tfr cells. J Allergy Clin Immunol. 2018;141(2):775-777.e6.

36. Wing JB, Ise W, Kurosaki T, Sakaguchi S. Regulatory $\mathrm{T}$ cells control antigen-specific expansion of Tfh cell number and humoral immune responses via the coreceptor CTLA-4. Immunity. 2014;41(6):1013-1025.

37. Shrestha S, Yang K, Guy C, Vogel P, Neale G, Chi $H$. Treg cells require the phosphatase PTEN to restrain TH1 and TFH cell responses. Nat Immunol. 2015;16(2):178-187.

38. Brandt EB, et al. Targeting IL-4/IL-13 signaling to alleviate oral allergen-induced diarrhea. J Allergy Clin Immunol. 2009;123(1):53-58.

39. Cretney E, et al. Characterization of Blimp-1 function in effector regulatory T cells. J Autoimmun. 2018;91:73-82.

40. Cretney E, et al. The transcription factors Blimp-1 and IRF 4 jointly control the differentiation and function of effector regulatory T cells. Nat Immunol. 2011;12(4):304-311.

41. Yang G, et al. Transcriptional repressor Blimp1 regulates follicular regulatory T-cell homeostasis and function. Immunology. 2018;153(1):105-117.

42. Clement RL, et al. Follicular regulatory $\mathrm{T}$ cells control humoral and allergic immunity by restraining early B cell responses. Nat Immunol. 2019;20(10):1360-1371.

43. Cañete PF, et al. Regulatory roles of IL-10-producing human follicular T cells. J Exp Med. 2019;216(8):1843-1856.

44. Sayin I, et al. Spatial distribution and function of $\mathrm{T}$ follicular regulatory cells in human lymph nodes. J Exp Med. 2018;215(6):1531-1542.

45. Wing JB, et al. A distinct subpopulation of CD25- T-follicular regulatory cells localizes in the germinal centers. Proc Natl Acad Sci USA. 2017;114(31):E6400-E6409.

46. Fonseca VR, et al. Human blood $\mathrm{T}_{\mathrm{fr}}$ cells are indicators of ongoing humoral activity not fully licensed with suppressive function. Sci Immunol. 2017;2(14):eaan1487.

47. Liu C, Wang D, Song Y, Lu S, Zhao J, Wang H.
Increased circulating $\mathrm{CD} 4{ }^{+} \mathrm{CXCR} 5{ }^{+} \mathrm{FoxP}^{+}$follicular regulatory $\mathrm{T}$ cells correlated with severity of systemic lupus erythematosus patients. Int Immunopharmacol. 2018;56:261-268.

48. Yao Y, et al. Allergen immunotherapy improves defective follicular regulatory $\mathrm{T}$ cells in patients with allergic rhinitis. J Allergy Clin Immunol. 2019;144(1):118-128.

49. Haniuda K, Fukao S, Kodama T, Hasegawa H, Kitamura D. Autonomous membrane IgE signaling prevents IgE-memory formation. Nat Immunol. 2016;17(9):1109-1117.

50. Vanshylla K, Opazo F, Gronke K, Wienands J, Engels $\mathrm{N}$. The extracellular membrane-proximal domain of membrane-bound IgE restricts $B$ cell activation by limiting $\mathrm{B}$ cell antigen receptor surface expression. Eur JImmunol. 2018;48(3):441-453.

51. Yang Z, et al. Regulation of B cell fate by chronic activity of the IgE B cell receptor. Elife. 2016;5:e21238.

52. Brachs S, et al. Swiprosin-1/EFhd2 limits germinal center responses and humoral type $2 \mathrm{immu-}$ nity. Eur J Immunol. 2014;44(11):3206-3219.

53. Kerfoot SM, et al. Germinal center B cell and $\mathrm{T}$ follicular helper cell development initiates in the interfollicular zone. Immunity. 2011;34(6):947-960.

54. Choi YS, et al. ICOS receptor instructs T follicular helper cell versus effector cell differentiation via induction of the transcriptional repressor Bcl6. Immunity. 2011;34(6):932-946.

55. Gowthaman U, et al. Identification of a $\mathrm{T}$ follicular helper cell subset that drives anaphylactic IgE. Science. 2019;365(6456):eaaw6433.

56. Shen E, et al. Control of germinal center localization and lineage stability of follicular regulatory T cells by the Blimp1 transcription factor. Cell Rep. 2019;29(7):1848-1861.e6.

57. Turqueti-Neves A, et al. B-cell-intrinsic STAT6 signaling controls germinal center formation. Eur JImmunol. 2014;44(7):2130-2138.

58. Guthmiller JJ, Graham AC, Zander RA, Pope RL, Butler NS. Cutting edge: IL-10 is essential for the generation of germinal center $\mathrm{B}$ cell responses and anti-plasmodium humoral immunity. JImmunol. 2017;198(2):617-622.

59. Liu T, et al. PD-1 deficiency promotes TFH cells expansion in ITV-immunized mice by upregulating cytokines secretion. Parasit Vectors. 2018;11(1):397.
60. Caielli S, et al. A CD $4^{+} \mathrm{T}$ cell population expanded in lupus blood provides B cell help through interleukin-10 and succinate. Nat Med. 2019;25(1):75-81.

61. O'Garra A, Barrat FJ, Castro AG, Vicari A, Hawrylowicz C. Strategies for use of IL-10 or its antagonists in human disease. Immunol Rev. 2008;223:114-131.

62. O'Garra A, Vieira PL, Vieira P, Goldfeld AE. IL-10-producing and naturally occurring CD4+ Tregs: limiting collateral damage. JClin Invest. 2004;114(10):1372-1378.

63. Ouyang W, Rutz S, Crellin NK, Valdez PA, Hymowitz SG. Regulation and functions of the IL-10 family of cytokines in inflammation and disease. Annu Rev Immunol. 2011;29:71-109.

64. van de Veen W, Stanic B, Wirz OF, Jansen K, Globinska A, Akdis M. Role of regulatory B cells in immune tolerance to allergens and beyond. JAllergy Clin Immunol. 2016;138(3):654-665.

65. Pellerin L, et al. Peanut-specific type 1 regulatory $\mathrm{T}$ cells induced in vitro from allergic subjects are functionally impaired. JAllergy Clin Immunol. 2018;141(1):202-213.e8.

66. Böhm L, et al. IL-10 and regulatory T cells cooperate in allergen-specific immunotherapy to ameliorate allergic asthma. J Immunol. 2015;194(3):887-897.

67. Matsuda M, et al. Phenotype analyses of IL-10-producing Foxp $3{ }^{-} \mathrm{CD} 4^{+} \mathrm{T}$ cells increased by subcutaneous immunotherapy in allergic airway inflammation. Int Immunopharmacol. 2018;61:297-305.

68. Mocellin S, Marincola F, Rossi CR, Nitti D, Lise $\mathrm{M}$. The multifaceted relationship between IL-10 and adaptive immunity: putting together the pieces of a puzzle. Cytokine Growth Factor Rev. 2004;15(1):61-76.

69. Zennaro D, et al. Proteomics plus genomics approaches in primary immunodeficiency: the case of immune dysregulation, polyendocrinopathy, enteropathy, X-linked (IPEX) syndrome. Clin Exp Immunol. 2012;167(1):120-128.

70. Rao A, et al. Successful bone marrow transplantation for IPEX syndrome after reduced-intensity conditioning. Blood. 2007;109(1):383-385.

71. Halabi-Tawil M, et al. Cutaneous manifestations of immune dysregulation, polyendocrinopathy, enteropathy, X-linked (IPEX) syndrome. $\mathrm{Br} \mathrm{J}$ Dermatol. 2009;160(3):645-651. 